\title{
Transcriptomic Analysis of Arabidopsis Seedlings in Response to an Agrobacterium-Mediated Transformation Process
}

\author{
Kaixuan Duan, ${ }^{1}$ Christopher J. Willig, ${ }^{1}$ Joann R. De Tar, ${ }^{1}$ William G. Spollen, ${ }^{2}$ and Zhanyuan J. Zhang ${ }^{1, \dagger}$ \\ ${ }^{1}$ Plant Transformation Core Facility, Division of Plant Sciences, University of Missouri, Columbia, MO, U.S.A.; and ${ }^{2}$ Informatics \\ Research Core Facility, University of Missouri
}

Accepted 20 November 2017.

\begin{abstract}
Agrobacterium tumefaciens is a plant pathogen that causes crown gall disease. This pathogen is capable of transferring the T-DNA from its Ti plasmid to the host cell and, then, integrating it into the host genome. To date, this genetic transformation ability has been harnessed as the dominant technology to produce genetically modified plants for both basic research and crop biotechnological applications. However, little is known about the interaction between Agrobacterium tumefaciens and host plants, especially the host responses to Agrobacterium infection and its associated factors. We employed RNA-seq to follow the time course of gene expression in Arabidopsis seedlings infected with either an avirulent or a virulent Agrobacterium strain. Gene Ontology analysis indicated many biological processes were involved in the Agrobacterium-mediated transformation process, including hormone signaling, defense response, cellular biosynthesis, and nucleic acid metabolism. RNAseq and quantitative reverse transcription-polymerase chain reaction results indicated that expression of genes involved in host plant growth and development were repressed but those involved in defense response were induced by Agrobacterium tumefaciens. Further analysis of the responses of transgenic Arabidopsis lines constitutively expressing either the VirE2 or VirE3 protein suggested Vir proteins act to enhance plant defense responses in addition to their known roles facilitating T-DNA transformation.
\end{abstract}

Agrobacterium tumefaciens is a gram-negative soil-borne bacterium that is a known plant pathogen because it causes crown gall disease (Smith and Townsend 1907). This bacterium mobilizes a DNA segment, which is referred to as transfer DNA (T-DNA), from its tumor-inducing (Ti) plasmid to plant cells following the infection of a host species. Integration of T-DNA into the plant genome and its subsequent expression leads to the formation of tumorous growths that are characteristic of crown gall disease (Escobar and Dandekar 2003). By taking advantage of this unique ability to transmit DNA sequences to eukaryotes, Agrobacterium spp. are widely utilized in the development of transgenic plants for

${ }^{\dagger}$ Corresponding author: Zhanyuan J. Zhang;

E-mail: zhangzh@missouri.edu

Funding: This research is funded by University of Missouri Research Board and Life Sciences Fellowships.

*The $\boldsymbol{e}$-Xtra logo stands for "electronic extra" and indicates that five supplementary figures and two supplementary tables are published online.

@ 2018 The American Phytopathological Society crop breeding and basic research through a method known as the Agrobacterium-mediated transformation (Azpiroz-Leehan and Feldmann 1997).

Agrobacterium tumefaciens is the only phytopathogen that uses a type IV secretion system (Christie and Vogel 2000). In addition to the T-DNA segment, many virulence (Vir) proteins, including VirD2, VirE2, VirE3, VirD5 and VirF, are transferred to the plant cell through this system (Schrammeijer et al. 2003; Vergunst et al. 2000). The molecular roles that many of these Vir proteins play in the transformation process has been wellcharacterized. Inside the Agrobacterium tumefaciens cell, VirD2 functions as an endonuclease nicking the Ti plasmid near the border of the T-DNA sequence, which leads to the release of a linear single-stranded DNA (ssDNA) segment (Mysore et al. 1998). VirD2 is then covalently bound to the $5^{\prime}$ end of the T-strand and both are cotransferred into the host cell (Cascales and Christie 2004). VirE2 acts as a ssDNA binding protein (Vergunst et al. 2000). It has several functions in the plant cell during the transformation process. Once transported into plant cell cytoplasm, VirE2 molecules coat the T-strand to protect it from degradation by host plant nucleases (Citovsky et al. 1989). VirE2 contains nuclear localization signals within its structure, which helps to target the T-DNA to the plant cell nucleus (Citovsky et al. 1992, 1994; Gelvin 1998). In addition to binding the T-strand, VirE2 also interacts with a plant protein, VIP1 (VirE2-interacting protein 1), which further assists in targeting VirE2 to the nucleus to facilitate transformation (Li et al. 2005; Tzfira et al. 2001). VIP1 encodes a bZIP transcription factor that regulates stressresponsive gene expression (Pitzschke et al. 2009a and b). It has been hypothesized that Agrobacterium tumefaciens may hijack the VIP1 defense-signaling pathway for nuclear delivery of the TDNA complex, acting as a Trojan horse (Djamei et al. 2007). VirE3 is another translocated protein that has been shown to bind plant karyopherin $\alpha$ and interact with VirE2 to mimic VIP1 function, acting as an 'adapter' molecule between VirE2 and karyopherin $\alpha$ to assist VirE2 in targeting to the plant cell nucleus (Lacroix et al. 2005). In addition to binding VirE2, VirE3 is also a plant-active transcriptional activator, modulating plant gene expression to favor transformation (García-Rodríguez et al. 2006; Niu et al. 2015). VirF is an F-box protein that targets VIP1 and VirE2 for degradation, allowing the $\mathrm{T}$ complex to be uncoated prior to genomic integration and expression (Tzfira et al. 2004). However, the VirF protein on its own is unstable in plant cells, due to rapid degradation by the plant ubiquitin proteasome system (Magori and Citovsky 2011). To overcome this instability, agrobacteria transfer VirD5 to bind VirF inside the plant cell and prevent it from being rapidly degraded (Magori and Citovsky 2011). 
Throughout the interaction between agrobacteria and hosts, plant cells respond to the infection at different levels, from the outermost cell wall to the nucleus. Many plant proteins are known to be involved in this infection process (Ballas and Citovsky 1997; Deng et al. 1998; Hwang and Gelvin 2004). Like other bacterial pathogens, Agrobacterium infection triggers the host's innate immune response, characterized by the induction of defense-gene expression and reactive oxygen species (ROS) burst (Pitzschke et al. 2009a and b). This response is elicited by the perception of pathogen-associated molecular patterns (PAMPs) by specific plant receptors. A primary PAMP molecule originating from agrobacteria is elongation factor EF-Tu, which is perceived by the plant receptor EFR (Zipfel et al. 2006). The innate immune response triggered by Agrobacterium PAMP perception leads to major changes in plants, especially the reprogramming of gene expression. Using techniques such as cDNA amplified fragment length polymorphism, suppression subtractive hybridization, and DNA microarray analysis, many defense genes have been identified that were shown to be initially induced (Ditt et al. 2001, 2006; Veena et al. 2003) and then repressed by T-DNA and Vir proteins transferred at the later stages of transformation (Veena et al. 2003).

Although we have a relatively detailed understanding of the Agrobacterium-mediated transformation process, it is critically important to further understand the molecular mechanisms involved in Agrobacterium-plant interaction to apply this T-DNA transformation process to the benefit of crop breeding and basic research (Altpeter et al. 2016). There are known differences in infection between agrobacteria and other plant bacterial pathogens, such as the lack of hypersensitive response induction (Pu and Goodman 1993; Wood et al. 2001) and the delivery of several Vir proteins into plant cells. Thus, studying gene expression during the Agrobacterium infection process is a very important approach to elucidate molecular components of the interaction between agrobacteria and plants.

In this study, we used two Agrobacterium strains that differed in their ability to transfer Vir proteins and T-DNA to plant cells to infect whole Arabidopsis seedling plants. Using time course RNA-seq experiments to measure gene expression in these seedlings throughout infection, we identified plant genes that are differentially regulated during the infection process by these two Agrobacterium strains. This was followed by analyzing the responses of transgenic Arabidopsis lines constitutively expressing either the VirE2 or VirE3 proteins. The results suggested possible additional roles of Vir proteins in plant defense responses with important practical implication in addition to directly facilitating transformation.

\section{RESULTS}

\section{Evaluation of transformation efficiency.}

We employed two Agrobacterium strains (A136 and At804) (Veena et al. 2003) to infect very young Arabidopsis seedlings, using the AGROBEST assay (Wu et al. 2014). Both strains are disarmed, lacking oncogenes necessary for tumor formation, so there are no active plant hormones produced upon T-DNA transfer. Strain A136 lacks a Ti plasmid, making it avirulent and unable to transfer T-DNA and Vir proteins to plant cells. Strain At804 was derived from EHA105, which contains a disarmed super-virulent Ti plasmid and a binary vector, $\mathrm{pBISN} 1$ (Ni et al. 1995; Veena et al. 2003). This strain is virulent and able to transfer both T-DNA and Vir proteins into plant cells. pBISN1 has a gusA-intron gene, which is expressed only in plants but not in the agrobacteria ( $\mathrm{Ni}$ et al. 1995). To evaluate the transformation efficiency of the virulent strain used in this research in the AGROBEST infection system, At804 was used to infect 10day-old whole Arabidopsis seedlings. At 48 and $72 \mathrm{~h}$ postinfection (hpi), $\beta$-glucuronidase (GUS) activity was determined by histochemical GUS staining and methylumbelliferyl- $\beta$-glucuronide assay (Jefferson et al. 1987), to monitor transient gene expression as an indication of transient transformation efficiency. We observed a high frequency of transformation with strong GUS staining and high GUS enzymatic activity in Arabidopsis seedlings at $48 \mathrm{hpi}$ and even much stronger GUS activities at $72 \mathrm{hpi}$ (Fig. 1A and B). These results indicated that the T-DNA and Vir proteins were transferred into Arabidopsis cells at a high frequency even before the 48-hpi timepoint. In fact, in previous studies, the expression of genes encoded in T-DNA has been detected within $18 \mathrm{~h}$ and even as early as $6 \mathrm{~h}$ following infection (Narasimhulu et al. 1996; Veena et al. 2003). High frequency transformation ensures that RNA-seq reads from infected seedlings are a good representation of treatments with agrobacteria. Based on these results, we set up RNA-seq experiments using whole seedlings infected by either a virulent or an avirulent Agrobacterium strain and collected infected tissue samples at six different timepoints $(0,3,6,12,24$, and $48 \mathrm{hpi})$. In parallel, the mock inoculation (infection solution without Agrobacterium cells) was applied at each above timepoint as negative control. Total RNA samples extracted from seedling tissue samples collected for each treatment and mock control were submitted for sequencing.

\section{Time-course mRNA sequencing of Arabidopsis infected by Agrobacterium tumefaciens avirulent or virulent strains.}

After performing whole transcriptome sequencing and quality control treatment for all samples, the total number of reads recovered for each submitted sample averaged 19.7 million. After trimming and filtering the reads for base-call quality scores and adaptor sequences, over $95 \%$ of the reads remained in each sample (Supplementary Table S1). Of these filtered reads, less than $2 \%$ in each sample aligned to Arabidopsis thaliana mitochondrial DNA (mtDNA) sequences. In the TopHat alignment, greater than $95 \%$ of the set of filtered reads were mapped to the A. thaliana genome. Transcripts with very low abundance (less than 5 read counts aligning to them) were dropped from further analysis.

To understand the transcriptomic changes specifically induced by treatment with either an avirulent or virulent Agrobacterium strain, contrasts were made between samples from each pair of treatments at each individual timepoint contrasted with the $0-h$ control samples. Genes were considered significantly differentially expressed if they had a false discovery rate of less than 0.05 and showed at least a twofold change in expression between two treatments (Supplementary Fig. S1). Overall, there were 965 unique genes found to be differentially expressed, including all timepoints in the avirulent treatment over mock treatment (Fig. $1 \mathrm{C}$ and $\mathrm{D})$. Of these genes, 18 were differentially expressed at all timepoints $6 \mathrm{hpi}$ and later and 81 were present $12 \mathrm{hpi}$ and later, whereas 188 were present at both the 24- and 48-hpi timepoints (Fig. 1C and D). A total of 2,201 unique genes were found to be differentially expressed in the virulent treatment over mock treatment at all timepoints (Fig. 1C and D). Of these, 36 were differentially expressed at all timepoints after $6 \mathrm{~h}, 148$ were present at all comparisons after $12 \mathrm{~h}$, and 394 were differentially expressed at the 24- and 48-hpi timepoints together (Fig. 1C and D). A total of 890 genes were differentially expressed in both the avirulent to mock and virulent to mock comparisons (Fig. 1C and D).

We further plotted all genes with a transcript abundance of more than five read counts in at least five samples by their $\log _{2^{-}}$ transformed FPKM (fragments per kilobase of transcript per million mapped reads) values in each experimental treatment at each of the timepoints examined after $0 \mathrm{~h}$ (Supplementary Fig. $\mathrm{S} 2$ ). At the earliest stages of infection (3 and $6 \mathrm{hpi}$ ), the few genes that were considered differentially expressed between 
any two treatments had a high expression level in all treatments relative to the majority of differentially expressed genes (DEGs) at later timepoints. As the time after inoculation increased for both treatments, more genes were seen to have a higher expression relative to mock than those that had a lower expression level in both the virulent and avirulent treatments.

\section{Expression profiling.}

To group the DEGs based on their expression patterns in each treatment condition through time, we performed $k$-means clustering on a set of genes that included all that were shown to be significantly differentially expressed in any contrast treatments. The set was grouped into 16 clusters by utilizing a tree-cutting method. Clusters 3, 4, 7, 8, 9, 10, 13, and 15 (totaling 999 of the DEGs) showed a general pattern in which higher expression levels were seen over time for the avirulent and virulent treatments while levels were near constant or decreased over time in the mock treatment (Fig. 2). In these clusters, the avirulent and virulent treatments followed mostly similar profiles over time, with some divergence seen between them at the 24- and 48-hpi timepoints (Fig. 2). Clusters 2, 5, 6, 11, 12, 14, and 16 showed a profile of decreased expression levels in avirulent and virulent treatments over time, relative to mock, with the virulent treatment showing slightly decreased expression relative to avirulent (Fig. 2). Cluster 1 displayed a unique pattern in which expression levels increased in the mock treatment at 24 and $48 \mathrm{hpi}$, whereas levels stayed constant or decreased slightly in the other two treatments, indicating that avirulent and virulent strains may suppress expression of this group of genes (Fig. 2). This data displays the broad differences in expression patterns of DEGs seen during the infection process by the two different Agrobacterium strains.

\section{Identification of specific cellular and biological processes involved in Agrobacterium infection.}

To investigate some of the wide-scale changes in gene expression relating to specific cellular and biological processes, we employed Gene Ontology (GO) analysis to categorize separate sets of both upregulated and downregulated genes for each of the contrasting treatments made at each timepoint. Figure 3 shows the degree to which selected GO biological process categories were overrepresented among the DEGs at each

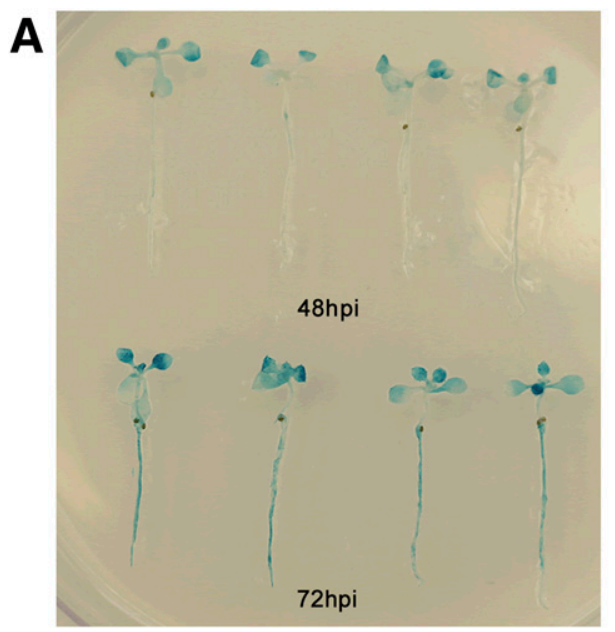

B
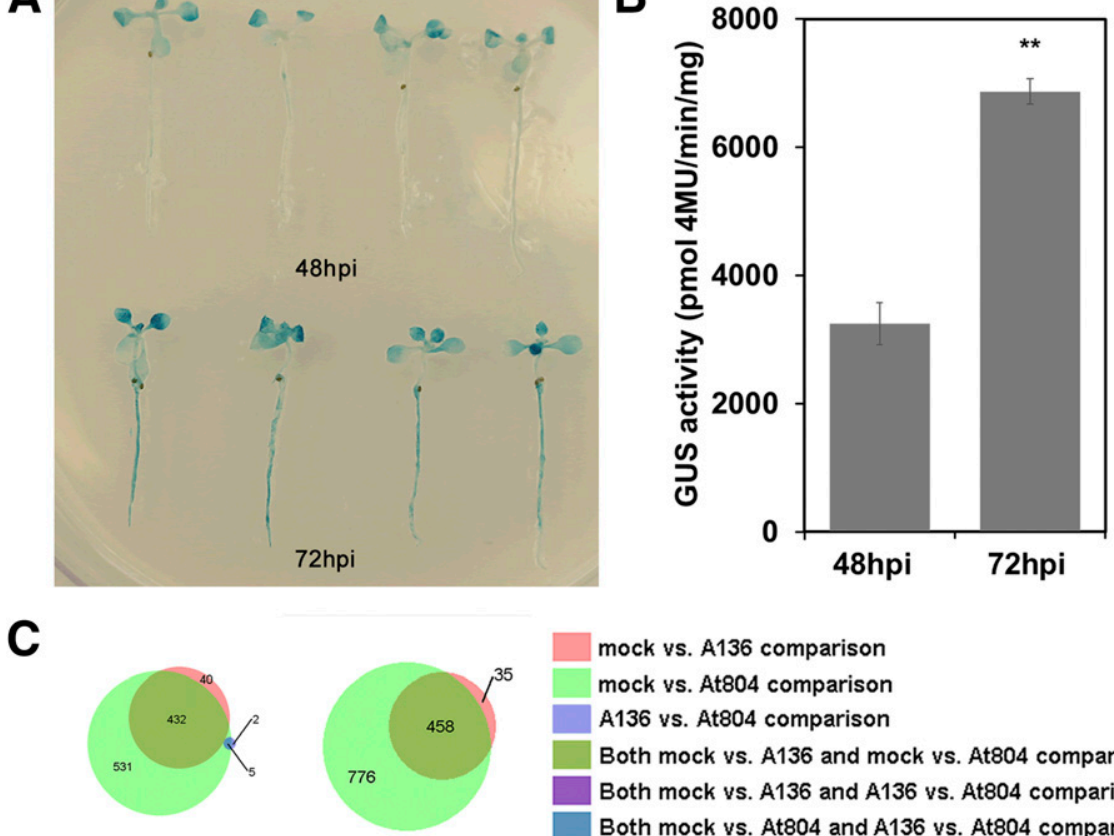

mock vs. A136 comparison

mock vs. At804 comparison

A136 vs. At80 4 comparison

Both mock vs. A136 and mock vs. At804 comparisons

Both mock vs. A136 and A136 vs. At804 comparions

Both mock vs. At804 and A136 vs. At804 comparisons

All three comparisons

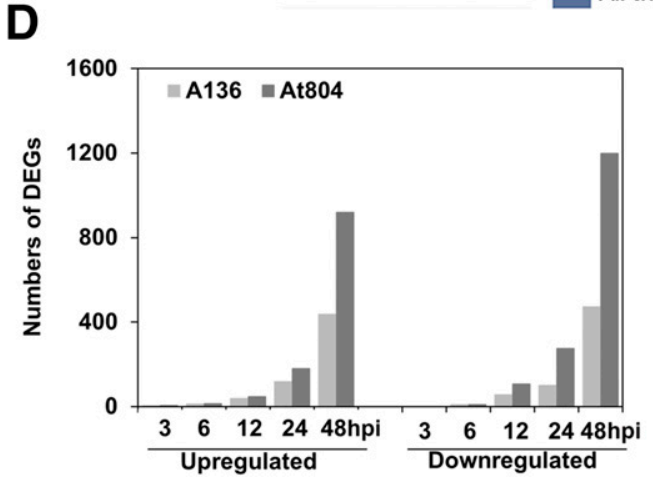

Fig. 1. Evaluation of Agrobacterium-mediated transformation efficiency and an overview of differentially expressed genes (DEGs). A, Histochemical $\beta$ glucuronidase (GUS) staining of Arabidopsis whole seedlings at 48 and $72 \mathrm{~h}$ postinfection (hpi) (upper and lower panels, respectively). B, Methylumbelliferyl$\beta$-glucuronide assay with significant difference $(P<0.01)$ in activity indicated by asterisks $(* *)$. The AGROBEST assay (Wu et al. 2014) was employed to transform 10-day-old whole Arabidopsis seedlings using strain At804. Transformation efficiency was evaluated at 48 and 72 hpi. C, Venn diagram displaying sets of both upregulated (on the left) or downregulated genes (on the right) unique to each comparison or shared between comparisons for treatment with strains A136 and At804 at all timepoints combined. D, Total counts of genes found to be up- or downregulated upon infection with strains A136 or At804 compared with the mock treatment at each timepoint. DEGs were from infection by different Agrobacterium strains versus the mock treatment. 
timepoint. Categories such as 'defense response', 'response to stress', 'response to reactive oxygen species', 'cellular response to decreased oxygen levels', and 'response to wounding' are overrepresented among upregulated genes as early as the 12-hpi timepoint in both the avirulent over mock and the virulent over mock comparisons (Fig. 3A). By contrast, the categories 'cell wall organization or biogenesis', 'DNA replication', 'external encapsulating structure organization', and most of 'metabolic process' are all overrepresented among downregulated genes in both comparisons (Fig. 3B). This indicates that Agrobacterium infection generally induces plant stress and defense responses while repressing genes associated with normal plant growth.

We then used quantitative reverse transcription-polymerase chain reaction (qRT-PCR) to validate the RNA-seq expression results for genes related to different cellular and biological processes involved in Agrobacterium infection. At 1 g25260 is a ribosomal L10 family protein, which functions in translation as a component of the ribosome and, therefore, is important in plant growth and development. qRT-PCR results showed that this gene's expression increased over time in the mock treatment, peaking at $24 \mathrm{hpi}$, however, little change was observed for treatment by either Agrobacterium tumefaciens strain,
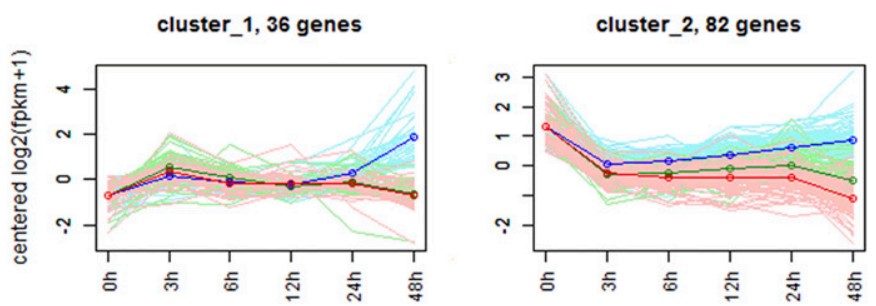

cluster_5, 143 genes

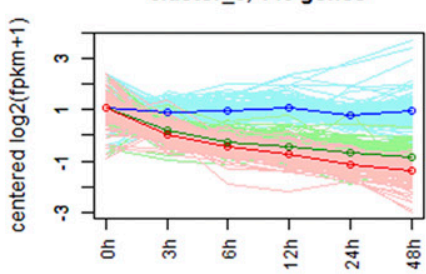

cluster_6, 116 genes

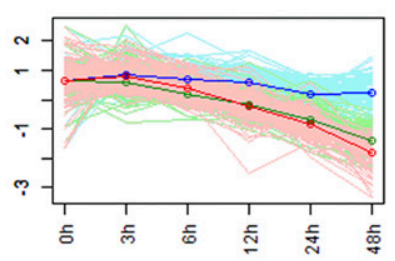

cluster_10, 68 genes

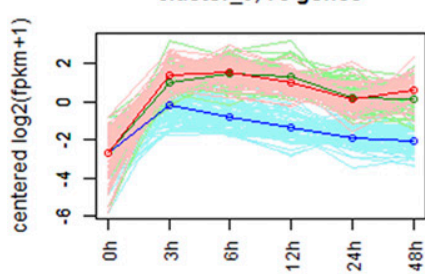

cluster_13, 25 genes
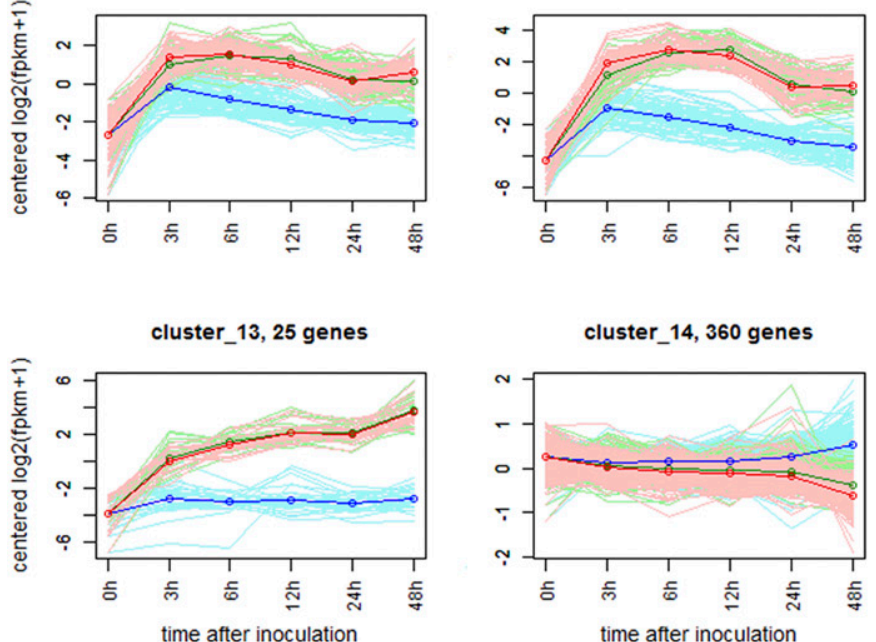

cluster_14, 360 genes

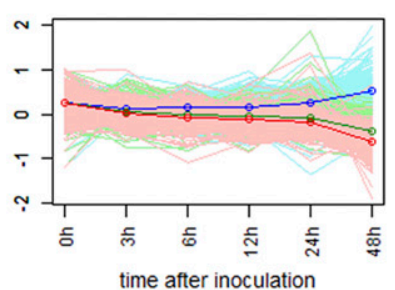

although there was a slight increase at the 3-hpi timepoint in the A136 treatment (Fig. 4A). Additional qRT-PCR results showed that closely related ribosomal genes followed a similar expression pattern throughoutthe infection process (Supplementary Fig. S3). For the cell-cycle gene $C Y C B 1 ; 1$, the expression level increased in mock treatment starting from $6 \mathrm{hpi}$, but there was no change in expression over infection time for treatment with either strain (Fig. 4B). Another group of genes that were investigated with qRT-PCR was the DNA methyl transferases. Our results showed that the expression of these genes also increased in the mock treatment over time, but showed no change over time for treatment with either strain (Fig. 4C). From our RNA-seq data, we found that the cell wall organization or biogenesis GO process was repressed by both Agrobacterium treatments. Therefore, we tested the expression of one gene related to this process, namely UGT74B1 (AT1G24100), with qRT-PCR. The result showed that this gene's expression increased in the mock treatment, while it decreased in the A136 and At804 treatments over infection time (Fig. 4D). We tested the expression levels of a few other genes related to plant growth and development using qRT-PCR, which followed the same trend of increased expression in the mock treatment, with few changes in either Agrobacterium treatment.
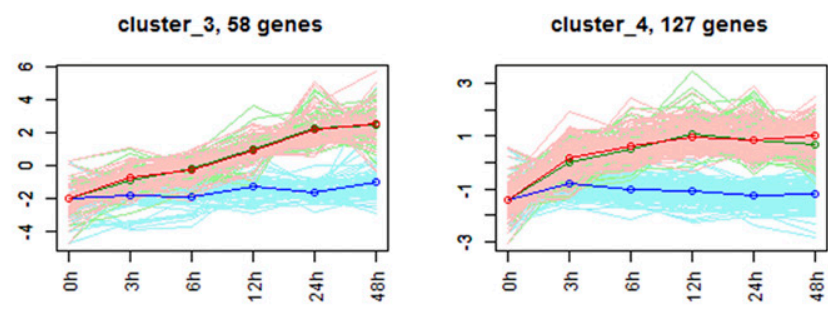

cluster 7,139 genes

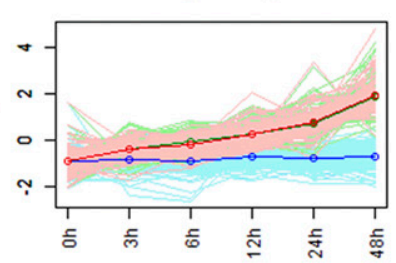

cluster_8, 90 genes

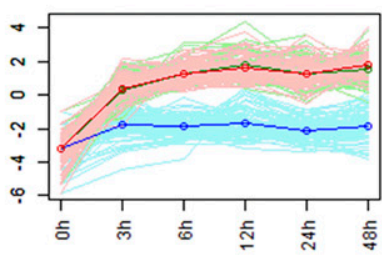

cluster_11, 263 genes

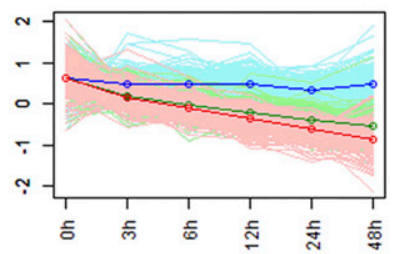

cluster_12, 204 genes cluster_15, 414 genes

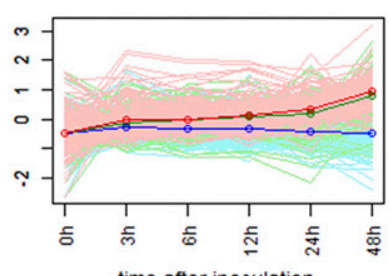

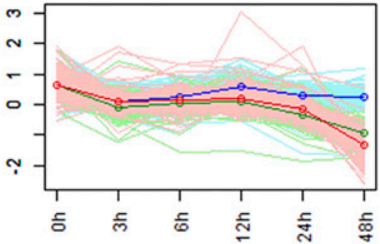

cluster_16, 73 genes

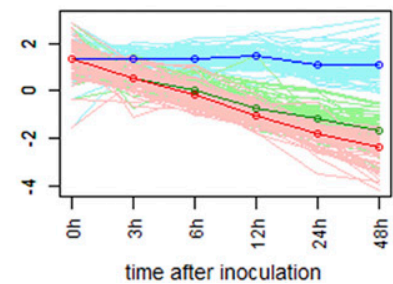

Fig. 2. $k$-means clustering by tree-cutting over a 48-h time course. A set of all differentially expressed genes (DEGs) (differentially expressed in any comparison) were clustered into 16 groups based on their overall expression profiles. The total number of DEGs falling into each cluster are indicated above each subplot. Blue, green, and red lines represent mock, avirulent (A136), and virulent (At804) Agrobacterium strain treatments, respectively. Bold, darkened lines in each color show the average gene expression profile for each treatment. 
In addition to genes relating to plant growth and development, we also validated the expression of defense responsive genes. Our qRT-PCR results showed that some defense responsive genes, such as FRK1, NHL10, PRX33, and ERF5, were strongly induced in both Agrobacterium treatments compared with mock (Fig. 4E to H). Other defense and stress responsive genes that were examined showed this same trend (Supplementary Fig. S4). Overall, the results of these qRT-PCR validation tests indicate that Agrobacterium tumefaciens represses plant growth and development and induces defense and stress responses.
Different expression patterns of plant genes in response to infection by different Agrobacterium strains.

A previous study has shown that transferred factors (T-DNA and Vir proteins) from Agrobacterium tumefaciens were responsible for the differential expression of some genes during the transformation process (Veena et al. 2003). They found that some defense-related genes were repressed, whereas other genes important for T-DNA integration were induced at later stages of the transformation process. Here, using AGROBEST as a different transformation system from Veena et al. (2003) and
A
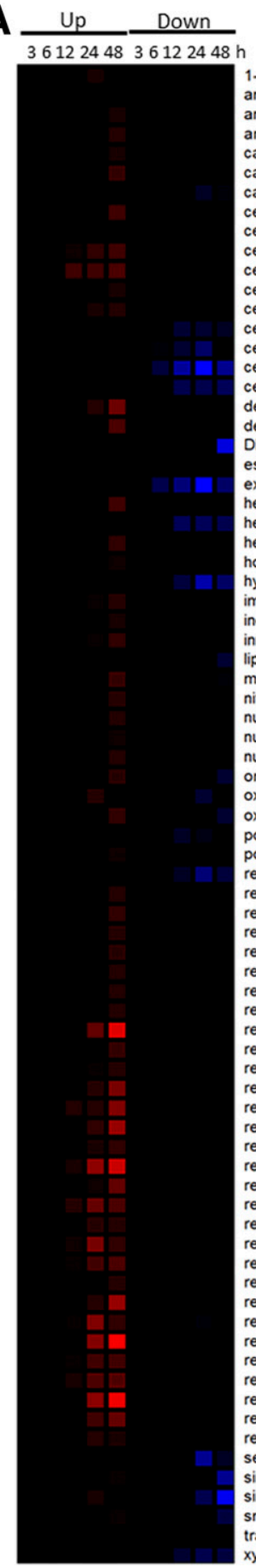

1-aminocyclopropane-1-carboxylate metabolic process (GO:0018871) amide biosynthetic process (GO:0043604) anaerobic respiration (GO:0009061) aromatic compound biosynthetic process (GO:0019438) camalexin biosynthetic process (GO:0010120) carboxylic acid metabolic process (GO:0019752) catabolic process (GO:0009056) cellular metabolic process (GO:0044237) cellular protein metabolic process (GO:0044267) cellular response to chemical stimulus (GO:0070887) cellular response to decreased oxygen levels (GO:0036294) cellular response to organic substance (GO:0071310) celllular response to organic substance (GO stimulus (GO:0051716) cell wall macromolecule metabolic process (GO:0044036) cell wall modification (GO:0042545) cell wall organization or biogenesis (GO:0071554) cell wall polysaccharide metabolic process (GO:0010383) defense response (GO:0006952) defense response (GO:0006952) defense response to bacterium DNA replication (GO:0006260) external encapsulating structure organization (GO:0045229) heat acclimation (GO:0010286) hemicellulose metabolic process (GO:0010410) heterocycle metabolic process (GO:0046483) hormone-mediated signaling pathway (GO:0009755) hydrogen peroxide catabolic process (GO:0042744) immune response (GO:0006955)

indole-containing compound metabolic process (GO:0042430) innate immune response (GO:0045087) lipid biosynthetic process (GO:0008610) monocarboxylic acid metabolic process (GO:0032787) nitrogen compound metabolic process (GO:0006807) nucleic acid-templated transcription (GO:0097659) nucleobase-containing compound biosynthetic process (GO:0034654) nucleobase-containing compound metabolic process (GO:0006139) organic acid metabolic process (GO:0006082)
oxidation-reduction process (GO:0055114) oxidation-reduction process (GO:0055114)
oxoacid metabolic process (GO:0043436) polysaccharide metabolic process (GO:0005976) positive regulation of cellular process (GO:0048522) reactive oxygen species metabolic process (GO:0072593) regulation of cellular macromolecule biosynthetic process (GO:2000112) regulation of cellular metabolic process (GO:0031323) regulation of cellular process (GO:0050794) regulation of gene expression (GO:0010468) regulation of nucleic acid-templated transcription (GO:1903506) regulation of primary metabolic process (GO:0080090) regulation of RNA metabolic process (GO:0051252)

response to abiotic stimulus (GO:0009628) response to abscisic acid (GO:0009737) response to biotic stimulus (GO:0009607) response to chitin (GO:0010200)

response to decreased oxygen levels (GO:0036293) response to decreased oxygen levels (GO:00362
response to endogenous stimulus (GO:0009719) response to external stimulus (GO:0009605) response to external stimulus (G)

response to heat (GO:0009408)

response to hormone (GO:0009725)

response to hydrogen peroxide (GO:0042542)

response to inorganic substance (GO:0010035)

response to light intensity (GO:0009642)

response to light stimulus (GO:0009416)

response to lipid (GO:0033993)

response to nitrogen compound (GO:1901698)

response to oxidative stress (GO:0006979)

response to oxygen-containing compound (GO:1901700)

response to radiation (GO:0009314)

response to reactive oxygen species (GO:0000302)

response to stress (GO:0006950)

response to temperature stimulus (GO:0009266)

response to wounding (GO:0009611)

secondary metabolic process (GO:0019748)

single-organism cellular process (GO:0044763)

single-organism cellular process (GO:0044763)

small molecule metabolic process (GO:0044281)

small molecule metabolic pr

xyloglucan metabolic process (GO:0010411)
B
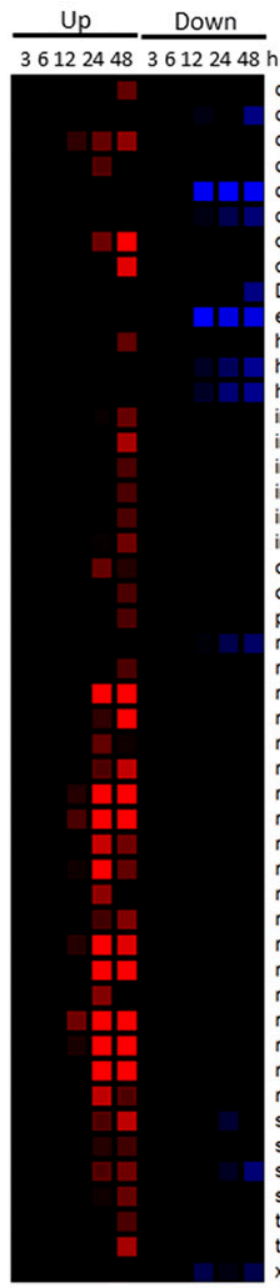

camalexin metabolic process (GO:0052317) carbohydrate metabolic process (GO:0005975)

cellular response to decreased oxygen levels (GO:0036294) cellular response to stress (GO:0033554)

cell wall organization or biogenesis (GO:0071554)

cell wall polysaccharide metabolic process (GO:0010383) defense response (GO:0006952) defense response to bacterium (GO:0042742) DNA replication (GO:0006260) external encapsulating structure organization (GO:0045229) heat acclimation (GO:0010286) hemicellulose metabolic process (GO:0010410) hydrogen peroxide catabolic process (GO:0042744) immune response (GO:0006955)

indole-containing compound metabolic process (GO:0042430) indole phytoalexin biosynthetic process (GO:0009700) indole phytoalexin metabolic process (GO:0046217) induced systemic resistance (GO:0009682) innate immune response (GO:0045087) oxidation-reduction process (GO:0055114) oxoacid metabolic process (GO:0043436) phytoalexin metabolic process (GO:0052314) reactive oxygen species metabolic process (GO:0072593) regulation of response to stress (GO:0080134) response to abiotic stimulus (GO:0009628) response to biotic stimulus (GO:0009607) response to chitin (GO:0010200)

response to external stimulus (GO:0009605) response to heat (GO:0009408)

response to hydrogen peroxide (GO:0042542) response to inorganic substance (GO:0010035 response to light intensity (GO:0009642) response to light stimulus (GO:0009416) response to nitrogen compound (GO:1901698) response to oxidative stress (GO:0006979) response to oxygen-containing compound (GO:1901700) response to radiation (GO:0009314) response to radiation (GO:0009314) response to stress (GO:0006950) response to temperature stimulus (GO:0009266) response to wounding (GO:0009611) secondary metabolic process (GO:0019748) secondary metabolite biosynthetic process (GO:0044550) single-organism metabolic process (GO:0044710) sulfur compound metabolic process (GO:0006790) toxin biosynthetic process (GO:0009403) toxin metabolic process (GO:0009404) xyloglucan metabolic process (GO:0010411)

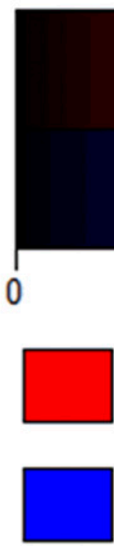

Fig. 3. Selected overrepresented Gene Ontology (GO) biological process categories across time course comparisons. Sets of genes used to determine significantly enriched GO terms were those found to be differentially expressed at a $P<0.1$ significance level and showed at least a twofold change in expression. The included categories are those that were found to be significantly enriched $(P<0.05)$ among either up- or downregulated genes at any timepoint in a given comparison. $P$ values from each category enrichment test have been negatively $\log _{10}$-transformed and plotted for both A, the avirulent (A136) treatment versus mock comparison and $\mathbf{B}$, the virulent (At804) treatment versus mock comparison. 
based on our RNA-seq data, we analyzed some of the differences in plant response to infection by these two Agrobacterium strains. To understand the transcriptomic changes specifically induced by treatment with the avirulent or the virulent Agrobacterium strain, contrasts were made between samples from each pair of treatments at each individual timepoint between avirulent and virulent treatment samples against the mock treatment. We found that 74 and 1,305 genes showed a specific response to the avirulent treatment and virulent treatment, respectively. Among the overrepresented GO categories unique to the upregulated genes in the avirulent over mock comparison (Fig. 5A) were the genes belonging to categories 'cellular
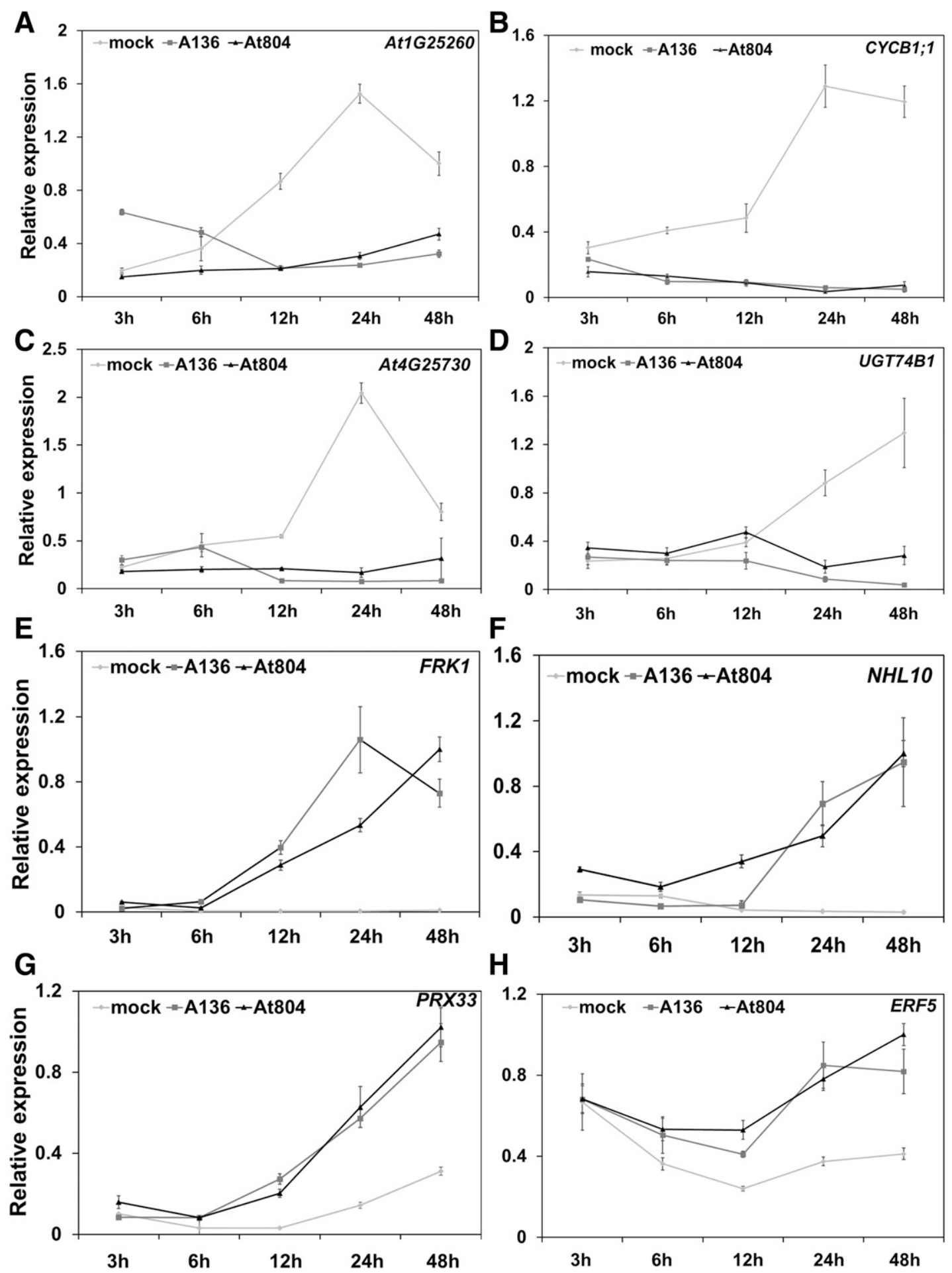

Fig. 4. Expression profiles of selected genes that showed a significant difference in transcriptional response through time upon infection by Agrobacterium strains (either A136 or At804) compared with mock infection, as validated by quantitative reverse transcription-polymerase chain reaction. Relative expression levels are shown for genes related to $\mathbf{A}$ to $\mathbf{D}$, growth and development and to $\mathbf{E}$ to $\mathbf{H}$, defense response. $\mathbf{A}$, Ribosomal protein L10 family protein homolog (At1g25260); B, Cyclin-dependent protein kinase CYCB1;1 (At4g37490); C, FtsJ-like methyltransferase family protein homolog (At4g25730); D, UDPGLUCOSYL TRANSFERASE 74B1, UGT74B1 (At1g24100); E, FLG22-INDUCED RECEPTOR-LIKE KINASE 1, FRK1 (At2g19190); F, NDR1/HIN1-LIKE, NHL10 (At2g35980); G, PEROXIDASE 33, PRX33 (At3g49110); and H, ETHYLENE RESPONSIVE ELEMENT BINDING FACTOR 5, ERF5 (At5g47230). Values represent the average of three replicates with error bars indicating standard deviation of the mean. 
response to stress' and 'secondary metabolite biosynthetic process', suggesting that the expression of these functional gene categories could be diminished by the treatment with the virulent strain over the avirulent strain. The presence of the 'secondary metabolite biosynthetic process' category among downregulated genes in the virulent treatment lends further credibility to this suggestion (Fig. 5A). There were several GO biological process categories that were overrepresented among upregulated genes in the virulent treatment that were not present among those of the avirulent treatment, such as 'ACC metabolic process', 'heterocycle metabolic process', 'response to abscisic acid', 'response to endogenous stimulus', and 'RNA metabolic process' (Fig. 5A). These terms show an overrepresentation beginning at the 24 or 48 -hpi timepoints, especially at $48 \mathrm{hpi}$. These indicate that some of the transcriptomic changes initiated by treatment with the Agrobacterium strain capable of delivering T-DNA along with Vir proteins into the plant cell may not be distinguishable from those by treatment with the avirulent strain until 24 to $48 \mathrm{~h}$ after inoculation or possibly even later.

We then plotted the expression levels (in FPKM) of individual DEGs falling into select GO categories onto a heat map with respect to time and treatment (Fig. 5B). For each of the categories, clear differences between expression levels in the mock treatment versus the other two treatments are readily evident. Although the differences in expression levels are not as evident on a broad scale between the virulent and avirulent treatments, some divergence in the expression of individual genes within particular GO categories can be seen in the 24- or 48-hpi samples, particularly among the 'secondary metabolite biosynthetic process' and 'response to abscisic acid' categories (Fig. 5B). We also found that some GO terms, such as 'metabolic process', 'response to stress', and 'regulation of cellular process' were suppressed in the virulent treatment as compared with the avirulent treatment. Another interesting observation was that there were some processes, such as 'defense response to bacteria', 'response to radiation', and 'innate immune response' that were overrepresented among the upregulated genes in both treatments, especially at the 24- or 48-hpi timepoints. These results indicate that not only does exposure to Agrobacterium cells induce plant response but, also, that the translocated T-DNA or Vir proteins trigger some unique responses in plant cells.

To investigate further the differences in the molecular response of plants to these two Agrobacterium strains, we selected some

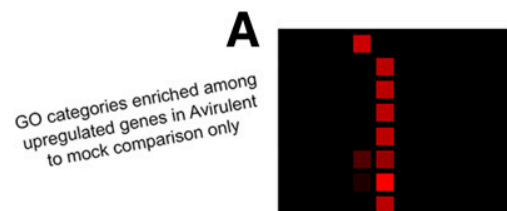

Cellular response to stress (GO:0033554) indole phytoalexin metabolic process (GO:0046217) induced systemic resistance (GO:0009682) phytoalexin metabolic process (GO:0052314) regulation of response to stress (GO:0080134) secondary metabolite biosynthetic process (GO:0044550) sulfur compound metabolic process (GO:0006790) oxin biosynthetic process (GO:0009403)

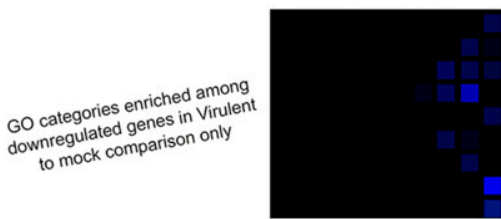

amide biosynthetic process (G0:0043604) catabolic process (GO:0009056) Clil macromolecule metabolic process (GO:0044036) cell wall modification (GO:0042545) Ipid biosynthetic process (GO:0008610) polysaccharide metabolic process (GO:0005976) secondary metabolite biosynthetic process (G0:0044550) ingle-organism cellular process (GO:0044763) mall molecule metabolic process (GO:0044281)

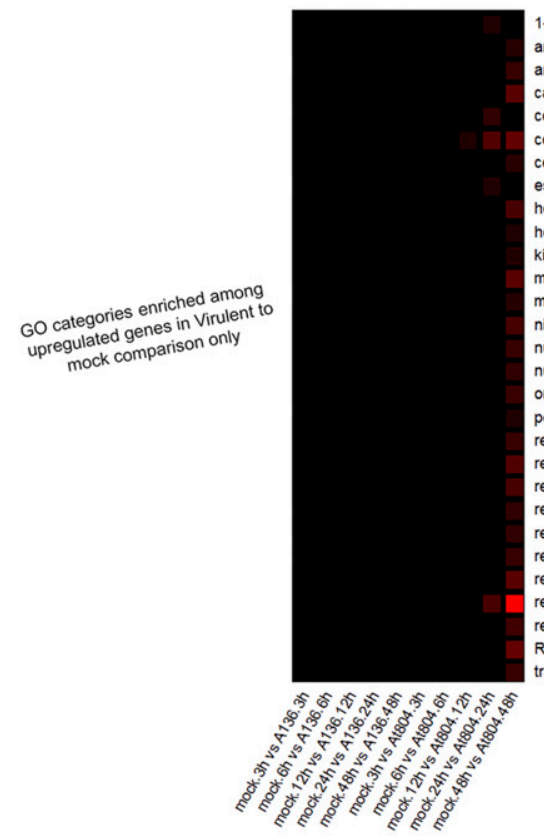

minocyclopropane-1-carboxylate aerobic respiration (GO:0009061) romatic compound biosynthetic process (GO:0019438) carboxylic acid metabolic process (GO:0019752) cellular protein metabolic process (GO:0044267) ellular response to chemical stimulus (GO:0070887) Cellular response to organic substance (GO:0071310) stablishment of localization (GO:0051234) heterocycle metabolic process (GO:0046483) ormone-mediated signaling pathway (GO:0009755) villing of cells of other organism (GO:0031640) monocarboxylic acid metabolic process (GO:0032787) mRNA splicing, via spliceosome (GO:0000398) nitrogen compound metabolic process (GO:0006807) ucleic acid-templated transcription (GO:0097659) ucleobase-containing compound metabolic process (GO:0006139) organic cyclic compound biosynthetic process (GO:1901362) positive regulation of cellular process (GO:0048522) regulation of cellular macromolecule biosynthetic process (GO:2000112) egulation of cellular metabolic process (GO:0031323) regulation of gene expression (GO:0010468) regulation of nucleic acid-templated transcription (GO:1903506) regulation of primary metabolic process (GO:0080090) regulation of RNA metabolic process (GO:0051252) esponse to abscisic acid (GO:0009737) response to endogenous stimulus (GO:0009719) response to lipid (GO:0033993) NNA metabolic process (GO:0016070) anscription, DNA-templated (GO:0006351)

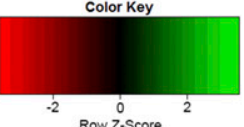

B

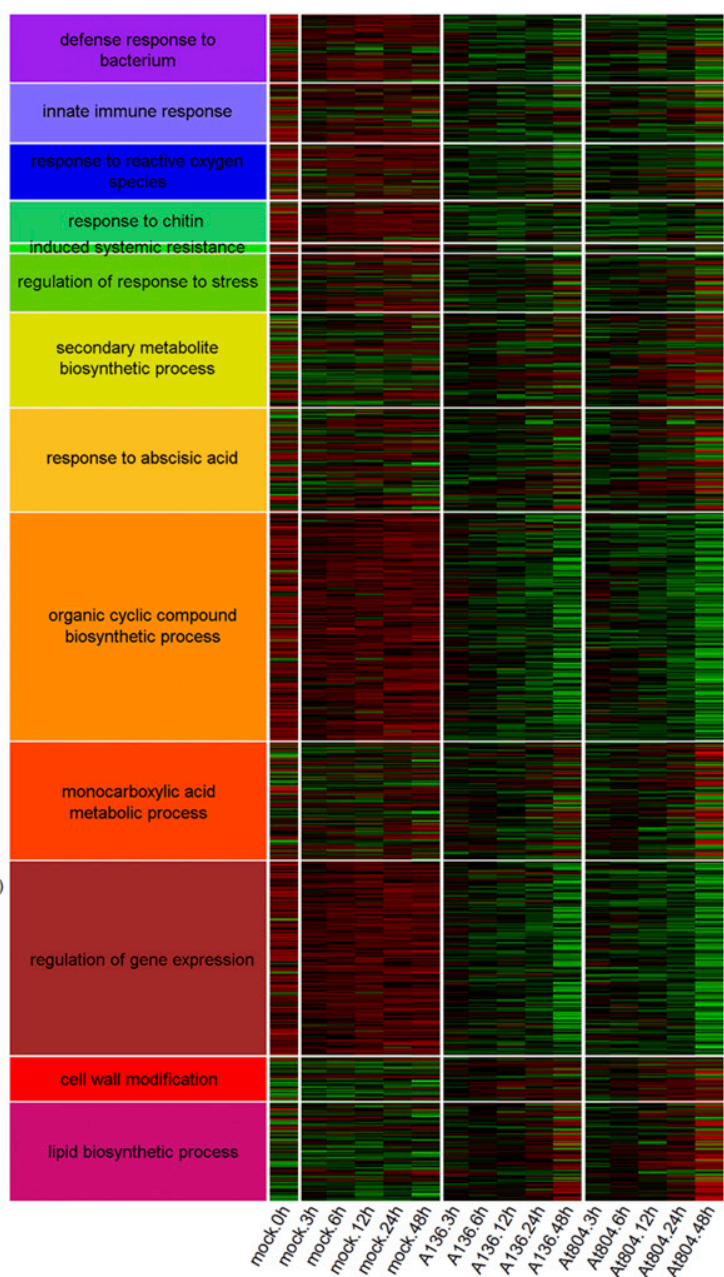

Fig. 5. Gene Ontology biological process categories in association with their gene expressions in heatmaps. A, Selected overrepresented Gene Ontology (GO) biological process categories unique to either avirulent (A136) to mock treatment or virulent (At804) to mock treatment contrasts. GO terms that showed enrichment at a significance level of $P<0.05$ among sets of either up- or downregulated genes that were unique to each contrast are shown. From the top, categories found to be significantly enriched among upregulated genes in the avirulent to mock comparison but not among those of the virulent to mock comparison are listed, followed by terms enriched among downregulated genes in the virulent to mock comparison only and, at the bottom, by those enriched among upregulated genes in the virulent to mock comparison only. There were no significantly enriched categories found that were unique to the avirulent to mock contrast among downregulated genes. B, Expression across all treatments among genes classified into selected GO biological process categories. Genes from 13 highlighted GO categories that were found to be differentially expressed $(P<0.05)$ were plotted by their expression intensities (in $\log _{2}$-transformed fragments per kilobase of transcript per million mapped reads values, zero-centered by row) for each treatment. 
individual genes involved in some of the previously highlighted GO biological process categories and used qRT-PCR to validate their expression levels as indicated from the RNA-seq data. One gene, At2g36770 from the UDP-glycosyltransferase superfamily, which is involved in the metabolic process category, was characterized. qRT-PCR results showed that, compared with the mock treatment, expression of this gene was induced by treatment with both Agrobacterium strains at the early stages of infection, but its expression level was much higher for A136 than for At804 treatment, especially at 3 and 6 hpi (Fig. 6A). Another gene, At5g52020, which is involved in the glucosinolate metabolic process, showed a similar expression pattern (Fig. 6C). The gene At $2 g 32020$, involved in plant stress response, was induced only at the early stages of treatment with A136 (Fig. 6B). Gene At2g31865, which is involved in cellular response to DNA damage stimulus, was found to be induced by treatment with either Agrobacterium strain at the 24-hpi timepoint but repressed at $48 \mathrm{hpi}$ in the At804 treatment (Fig. 6D). We also detected the induction of some defense-response genes during the infection process for treatment with both strains. Interestingly, the qRT-PCR results showed that, in comparisons of A136 and At804 treatments, the expression levels of defense genes such as PR2, RBOHD, ERF6, and At3g26680 were much higher in the At804 than in the A136 treatment, especially at 24 or 48 hpi (Fig. 6E to $\mathrm{H}$ ). These results further clarify some of the differences in plant response to treatment by avirulent or virulent Agrobacterium strains and highlight the response specific to the presence of transferred factors present in the virulent strain at the individual gene level.

\section{Constitutive expression of VirE2 or VirE3 in Arabidopsis regulates plant defense responses to agrobacteria.}

Since the translocated factors from the virulent Agrobacterium strain are able to trigger specific responses and stronger global defense responses than the avirulent strain in plant cells, we sought to investigate how the plant would respond to the individual Vir effector proteins, if any. VirE2 has been previously characterized as participating in an interaction with the plant protein VIP1, which is a transcription factor regulating defense and stress responses (Pitzschke et al. 2009a and b), while VirE3 has been shown to act as a plant-active transcriptional activator, for which the plant F-box protein VBF is a direct target (Niu et al. 2015). These two Vir proteins have the potential to modulate gene transcription through their previously established interactions with plant proteins. Therefore, we selected VirE2 and VirE3 for further study. First, we generated transgenic Arabidopsis lines that constitutively express $\operatorname{VirE2}(E 2-O E)$ and $\operatorname{VirE3}$ (E3-OE), respectively. We then confirmed overexpression of the transgenes in these lines using qRT-PCR (Fig. 7A). Because the physiological responses triggered by Agrobacterium infection in wild-type plants include ROS burst and induction of defense gene expression (Pitzschke et al. 2009a and b), we first tested the ROS burst through a luminol-based assay, using elf26 (an agrobacterium-derived PAMP) to trigger ROS burst in wild type as well as VirE2 and VirE3 constitutive expression lines. Our results showed that the photon emission elicited by elf 26 treatment over $40 \mathrm{~min}$ was clearly detectable in both wild type and VirE2 and VirE3 constitutive expression lines (Fig. 7B). However, there was a higher photon emission level observed for both VirE2 and VirE3 constitutive expression lines when compared with wild type (Fig. 7B).

Next, the wild type and transgenic lines were each treated with the avirulent Agrobacterium sp. strain A136 and the expression levels of some defense genes were examined using qRT-PCR. After 24 hpi, PR3, At4g00970, and At4g23260 were detected at higher expression levels in both VirE2 and VirE3 constitutive expression lines, while $F R K 1, P R 2$, and $P R 4$ were only found to be at a higher expression level in VirE3 constitutive expression lines (Fig. 7C to
H). Clearly, constitutive expression of VirE2 or VirE3 increases the plant defense responses to Agrobacterium infection. Interestingly, Niu et al. (2015) found that the plant genes involved in stress response, abiotic, and biotic stimuli were activated by VirE3, using Superviewer analysis in transgenic lines with inducible VirE3 expression. Using their sequencing data, we employed DAVID GO enrichment analysis to categorize, again, the biological process of upregulated and downregulated genes in the VirE3 expression lines. The results showed that, among upregulated genes, most were categorized into defense-response terms such as 'response to bacterium', 'response to chitin', 'response to salicylic acid', and more, while, among downregulated genes, there were no defense-responses processes that were significantly enriched (Supplementary Fig. S5). This strengthens the evidence for the role of VirE3 in increasing plant defense response.

\section{Constitutive expression of VirE2 or VirE3 in Arabidopsis decreases the efficiency of Agrobacterium-mediated transformation.}

An increase in plant defense response is usually accompanied by a decrease in bacterial infection. Since we have established that constitutive expression of VirE2 and VirE3 increases plant defense responses to agrobacteria, we wanted to know whether constitutive expression of either VirE2 or VirE3 also attenuated Agrobacterium-mediated transformation. To evaluate fully how such increased defense could affect transformation outcomes of various types of tissues, we employed three different transformation assays utilizing two different types of tissues. First, we evaluated the infection status at the whole-seedling level, using the AGROBEST infection method. Wild type (Col-0) as well as VirE2 and VirE3 constitutive expression lines (E2-OE and $E 3-O E$ ) were infected with the hypervirulent nontumorigenic Agrobacterium sp. strain GV3101 containing the binary vector pBISN1, which carries a GUS-intron gene. In wild-type plants, strong GUS staining and enzymatic activity were detectable at 3 dpi, while VirE2 or VirE3 constitutive expression lines exhibited only very weak GUS staining and activity (Fig. 8A and B). Then, a root transformation assay was used to evaluate the transformation efficiency in yet another assay system, as additional confirmation. Root segments taken from wild type as well as VirE2, and VirE3 constitutive expression lines were infected by GV3101 and transient gene expression was detected at 3 dpi. In wild-type roots, about $70 \%$ of the segments were transformed at a concentration of $10^{6} \mathrm{CFU} / \mathrm{ml}$ agrobacteria, while the VirE2 or VirE3 constitutive expression lines exhibited significantly decreased efficiency to about 40 to $50 \%$ at the same concentration (Fig. 8C and D). Similarly, at a concentration of $10^{7} \mathrm{CFU} / \mathrm{ml}$ agrobacteria, about $90 \%$ of the wild-type root segments showed GUS activity, whereas segments from VirE2 or VirE3 constitutive expression lines only had about $60 \%$ GUS activity (Fig. $8 \mathrm{C}$ and D). The efficiency of stable transformation was also tested in these lines by the induction of callus from the root segments at 3 weeks postinfection. The results showed that, at a concentration of $10^{6} \mathrm{CFU} / \mathrm{ml}$ agrobacteria, wild-type calli displayed intense GUS staining, with about $60 \%$ of segments showing GUS activity. This rate increased to about $65 \%$ at a $10^{7}-\mathrm{CFU} / \mathrm{ml}$ concentration. The percentage of calli with GUS activity in VirE2 or VirE3 constitutive expression lines was about half that of wild type for both agrobacteria concentrations (Fig. 8E and F). In fact, constitutive expression of wild type or truncated VirE2 in plants displaying resistance to Agrobacterium infection was reported in previous studies, explained by which redundant VirE2 proteins may compete less efficiently with T-complex (Citovsky et al. 1994; Krastanova et al. 2010). Together, our results support the idea that increased defense response due to the constitutive expression of VirE2 or VirE3 
reduces Agrobacterium-mediated transformation efficiency, regardless of different tissues to be transformed.

\section{DISCUSSION}

Although Agrobacterium tumefaciens has been widely used in plant transformation for decades, little is known about the involvement of plant factors in this process. In this report, we studied the differential expression of plant genes throughout the early stages of the Agrobacterium-mediated transformation process. We used both avirulent and virulent Agrobacterium strains to infect whole Arabidopsis seedlings and to analyze both plant responses to Agrobacterium colonization and specific responses to the transfer of T-DNA or Vir proteins into
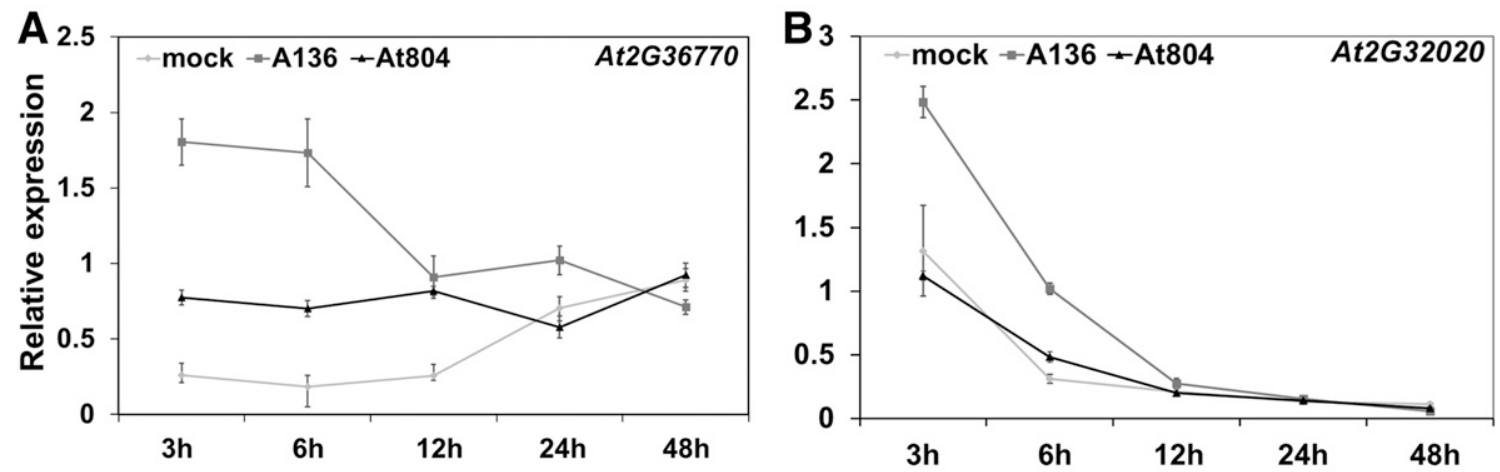

C

D
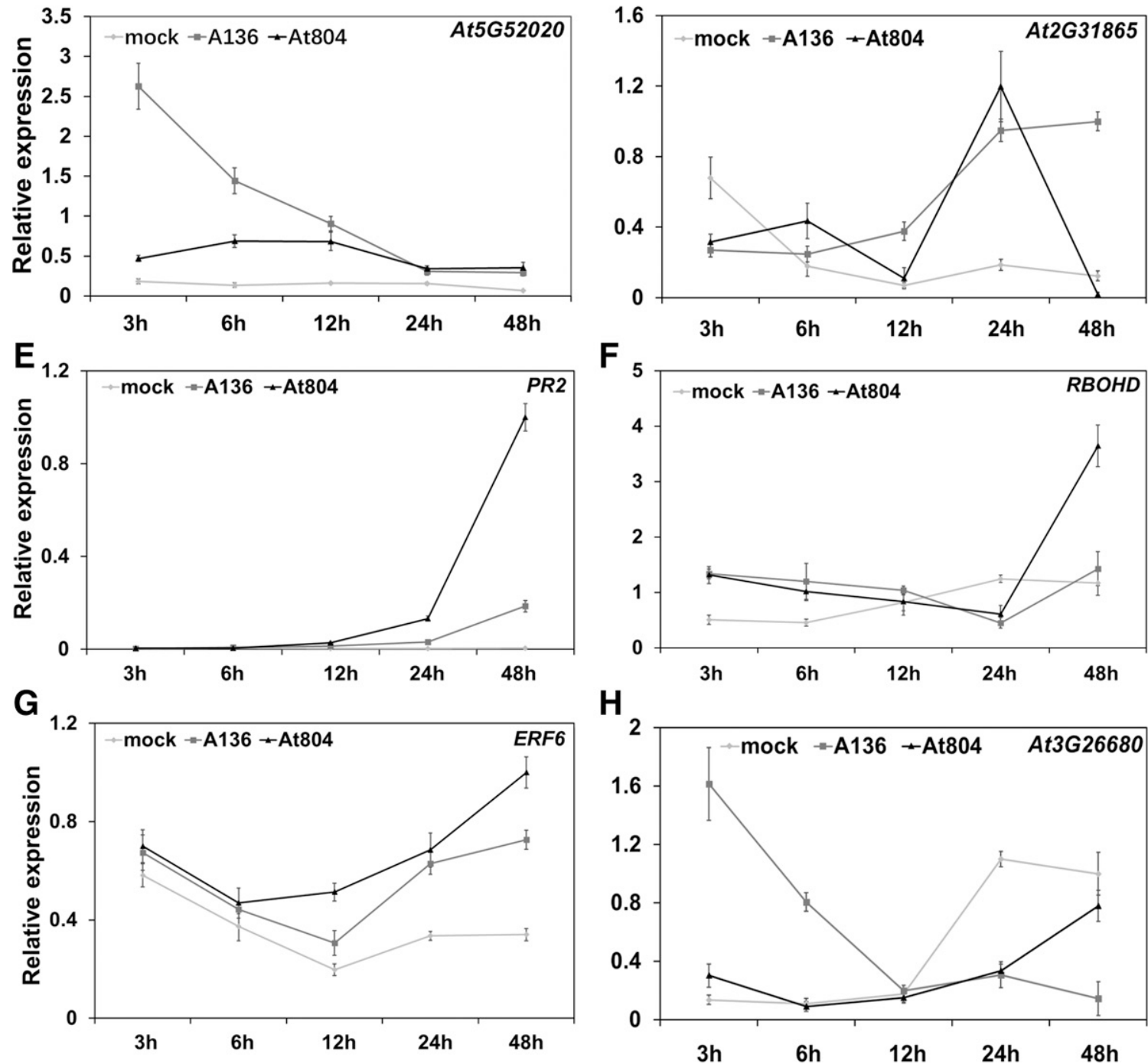

Fig. 6. Expression patterns of selected genes that were shown to differ in their response to treatment with the avirulent (A136) or virulent (At804) Agrobacterium strain, as evaluated by quantitative reverse transcription-polymerase chain reaction. Relative gene expression over the 48-h time course is shown for the following genes related to metabolism or defense. A, UDP-glycosyltransferase superfamily protein homolog (At2g36770); B, acyl-CoA N-acyltransferases (NAT) superfamily protein homolog (At2g32020); C, DREB subfamily A-4 of ERF/AP2 transcription factor family homolog (At5g52020); D, poly(ADP-ribose) glycohydrolase 2 (At2g31865); E, PR2 (At3g57260); F, RBOHD (At5g47910); G, ERF6 (At4g17490); and H, ATSNM1 (At3g26680, involved in a SNM-dependent recombinational repair process of oxidatively induced DNA damage). Values represent the average of three replicates, with error bars indicating standard deviation of the mean. 
plant cells. Using RNA-seq, we identified numerous DEGs during the initial stages of transformation.

The AGROBEST system employed in this study is based on Agrobacterium infection of the whole plant seedling whose various vegetative tissues are accessible to the Agrobacterium cells (Wu et al. 2014). This system is in sharp contrast to the Arabidopsis floral-dip method, which targets germline cells and is rarely employed in most plant transformation systems. Therefore, the RNA-seq results from AGROBEST are more representative of and, thus, more applicable to the real plant transformation practice in which vegetative tissues are typically deployed.

By using GO enrichment analysis, we determined that most of the genes identified as being repressed during the treatments by both avirulent and virulent Agrobacterium strains in this study were involved in plant growth and developmental processes such as cell-wall biogenesis and metabolic process. Selected genes classified into these categories, such as $C Y C B 1 ; 1$ and $U G T 74 B 1$, were shown to be repressed during Agrobacterium infection on an individual level (Figs. 3 and 4). This finding is
A

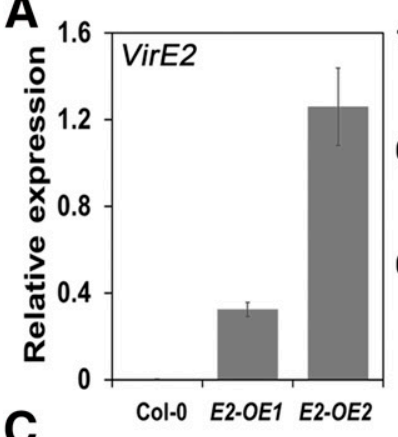

C

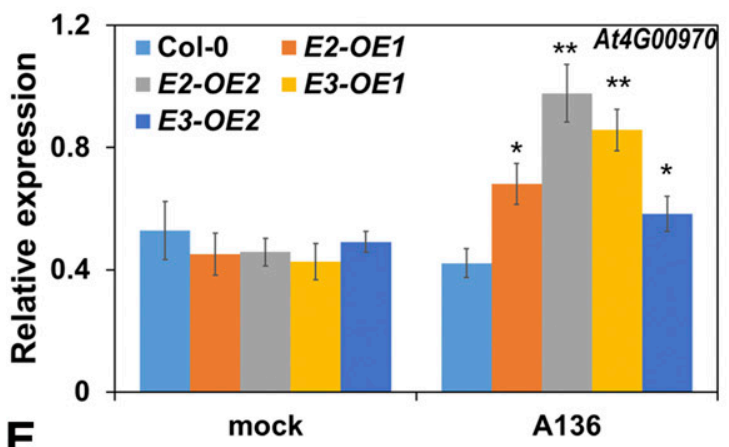

E
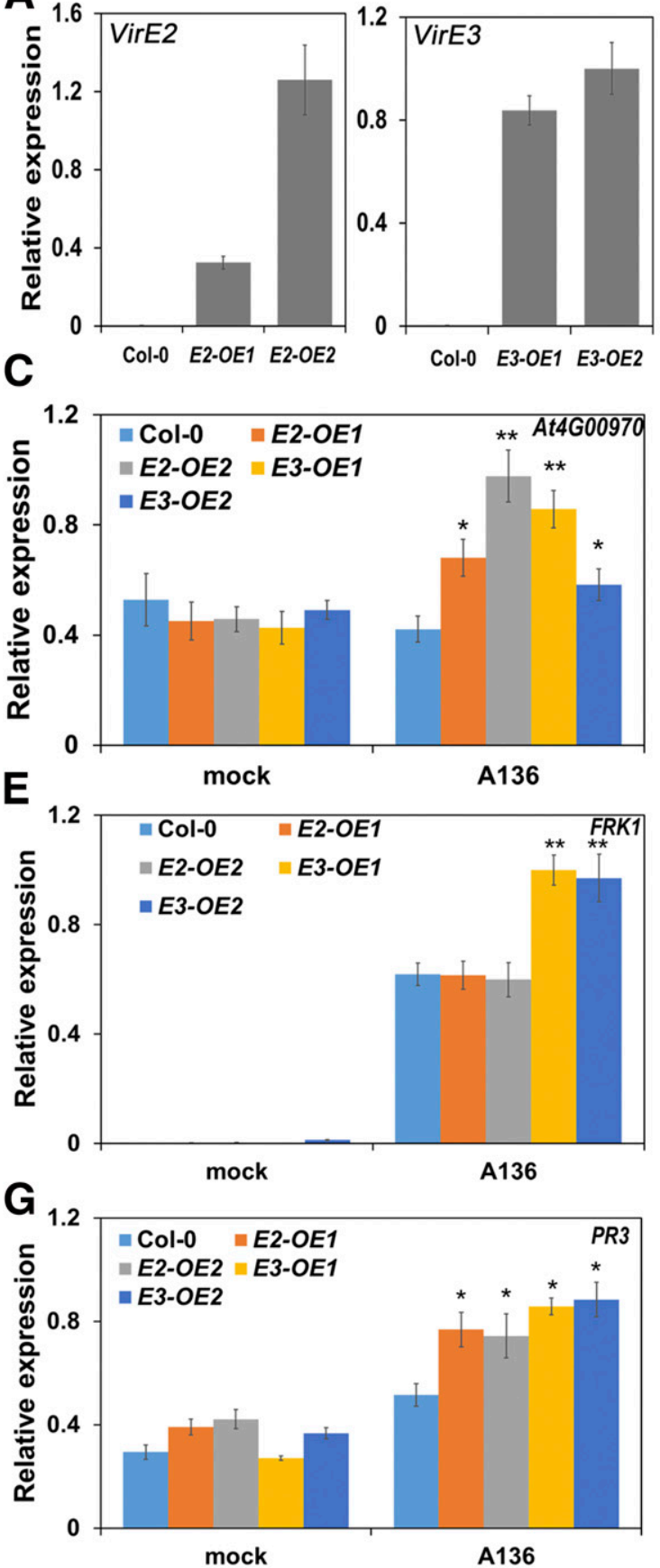

B
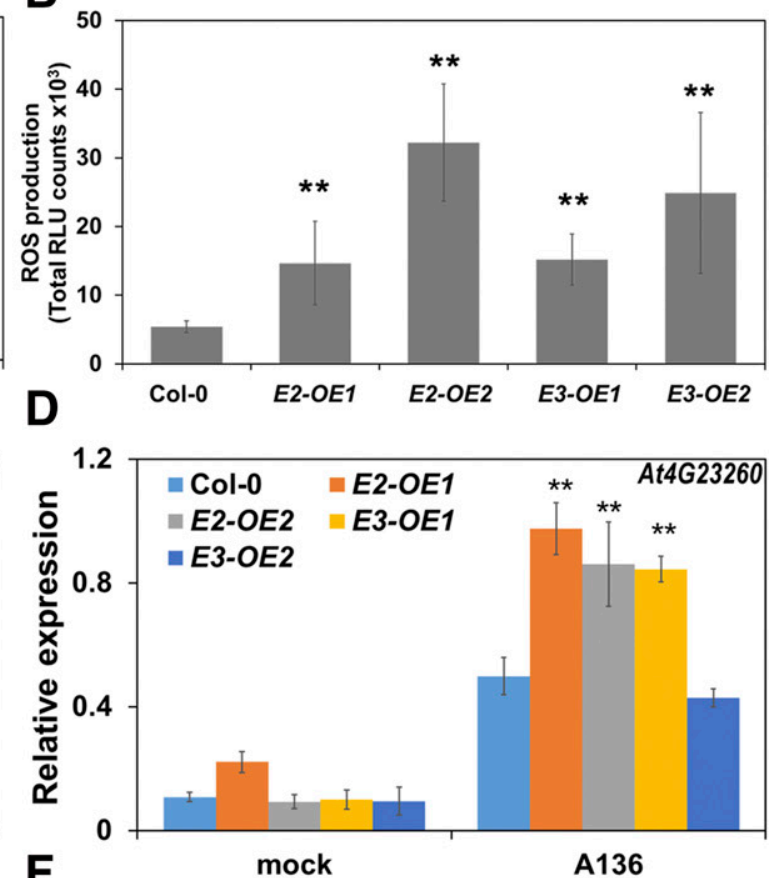

$\mathbf{F}$

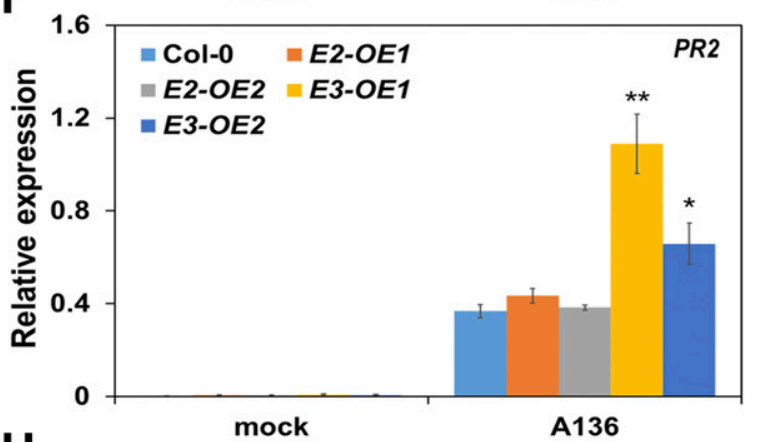

H

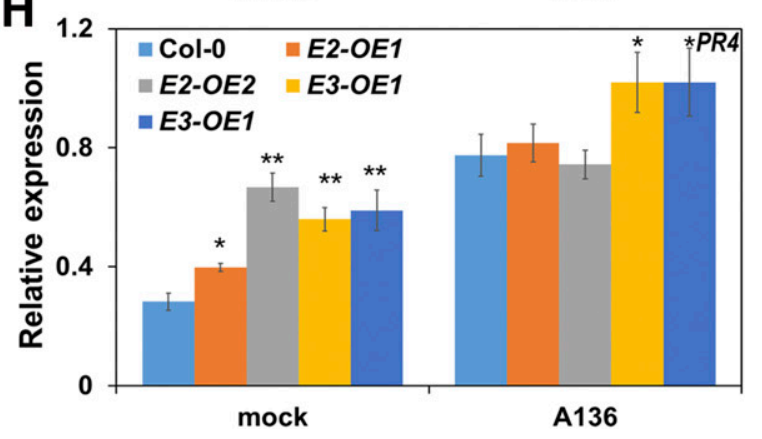

Fig. 7. Enhanced defense response of VirE2 and VirE3 constitutive expression lines (E2-OE and E3-OE lines) to Agrobacterium tumefaciens. A, Evaluation of VirE2 and VirE3 expression in Col-0 and transgenic plants by quantitative reverse transcription-polymerase chain reaction (qRT-PCR). B, Reactive oxygen species (ROS) production triggered by the pathogen-associated molecular pattern elf26 in Col-0 and $E 2-O E$ and $E 3-O E$ lines. ROS production is quantified as counts of total emitted photons during 40 min of treatment. $\mathbf{C}$ to H, qRT-PCR analysis of some defense gene expressions in Col-0 and $E 2-O E$ and $E 3-O E$ lines infected by Agrobacterium sp. strain A136 or mock-treated. Relative gene expression levels are shown for C and D, CYSTEINE-RICH RLK homologs (At4G00970, At4G23260), E, FRK1, F, PR2, G, PR3, and H, PR4. Values represent the average of three replicates, with error bars indicating standard deviation of the mean. One asterisk (*) indicates a significant difference at $P<0.05$, while two (**) indicate a significant difference at $P<0.01$. 
supported by the fact that plant growth and development can be restricted by using a high Agrobacterium concentration during infection. Too-high Agrobacterium concentrations have previously even been shown to cause an apoptotic response (Hansen 2000). This is the reason that antibiotics are commonly added to growth medium to eliminate agrobacteria after cocultivation in plant transformation attempts. Many genes induced by both avirulent and virulent Agrobacterium strains were found to be involved in defense and stress response. These genes are categorized under GO biological process terms such as 'response to chitin', 'response to biotic stimuli', 'response to abiotic stimuli' (Figs. 5 and 6). Ditt et al. (2001, 2006) and Veena et al. (2003) also found some defense genes that were regulated by Agrobacterium tumefaciens in their studies.
Previous studies have also shown that the Agrobacteriumderived EF-Tu peptide is an important PAMP, triggering a defense response through its interaction with the plant receptor EFR (Zipfel et al. 2006). All these findings help explain how agrobacteria can trigger the plant defense response.

By comparing the different gene expressions at different infection timepoints, we found differing responses of plants to Agrobacterium strains. In general, at the early stage of infection ( 3 to $6 \mathrm{hpi}$ ), there are fewer genes responding to Agrobacterium infection, and only some cell wall-related genes are involved in this early response (Figs. 1D and 6A and C). However, as the infection progresses (12 to $48 \mathrm{hpi}$ ), not only are more genes expressed but the gene expression levels also changed, especially for some genes in responses to abiotic and biotic stress as
A

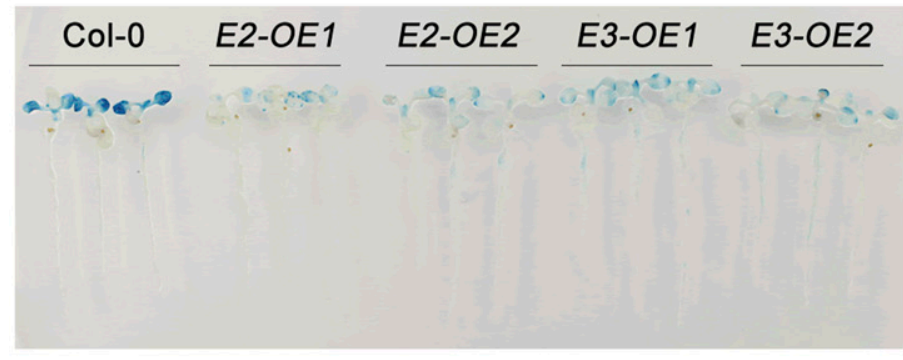

C

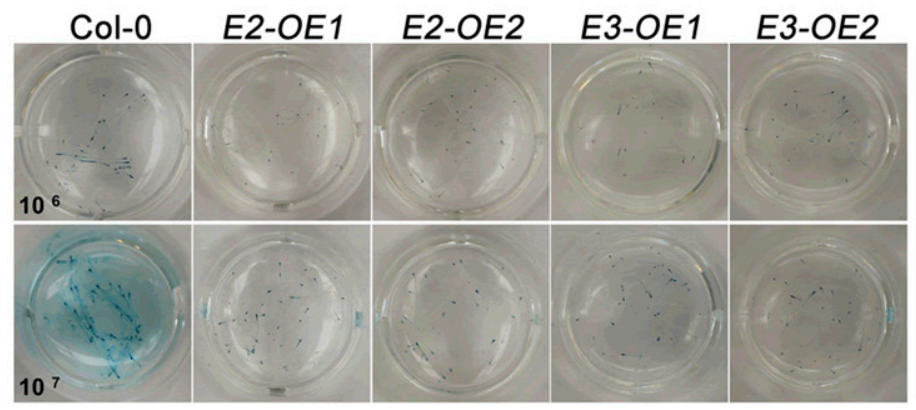

E

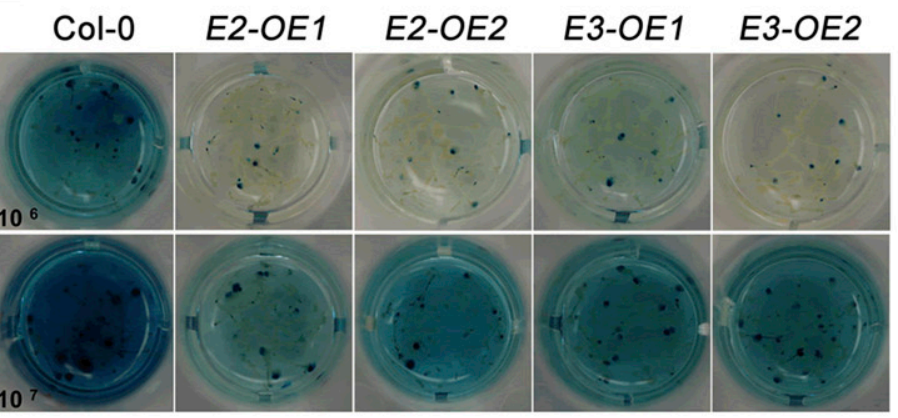

B

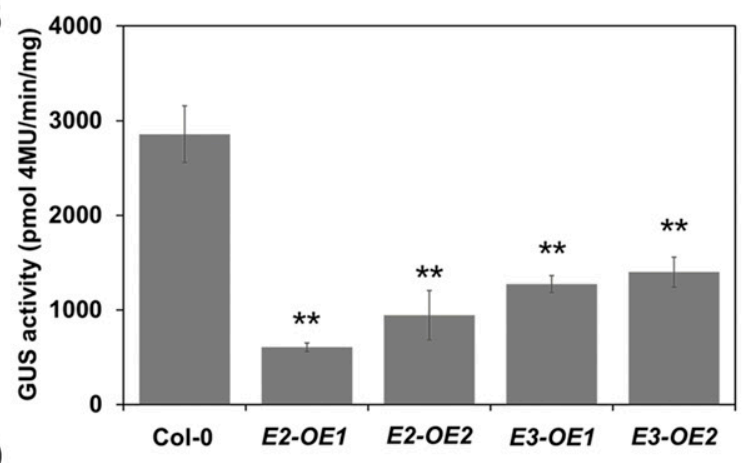

D

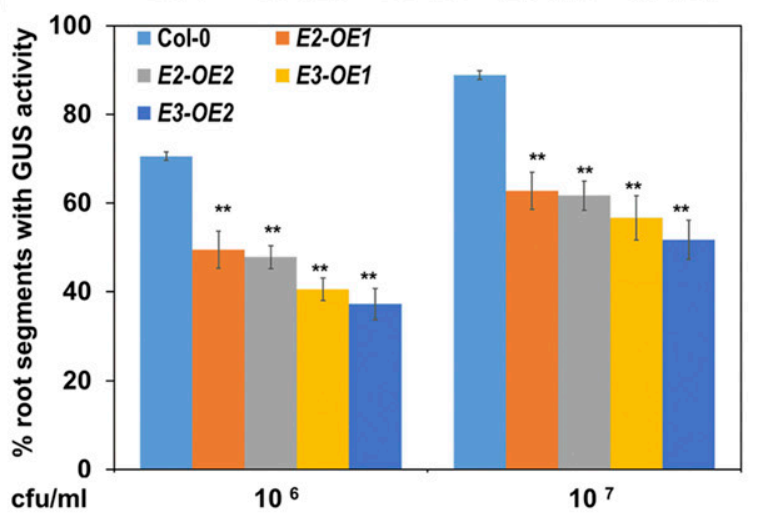

$\mathbf{F}$

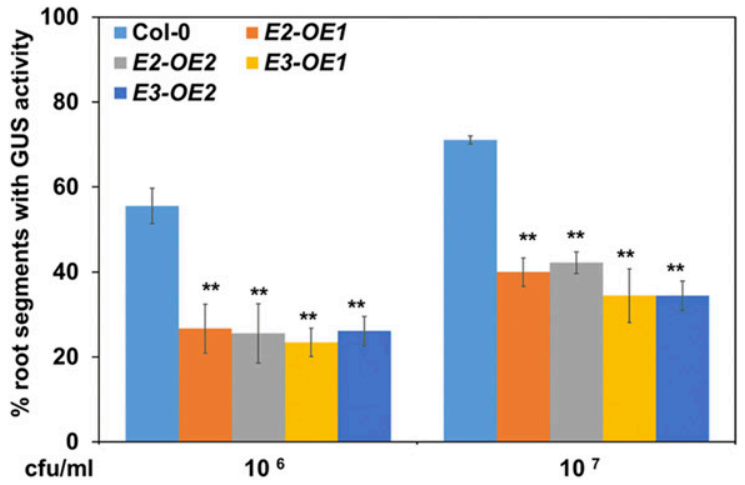

Fig. 8. Efficiency of Agrobacterium-mediated transformation in VirE2 and VirE3 constitutive expression lines (E2-OE and E3-OE lines) as compared with wild-type plants, using three different assays. A and B, Efficiency of transient transformation using AGROBEST assay (Wu et al. 2014). Ten-day-old Arabidopsis Col-0 and transgenic VirE2 and VirE3 constitutive expression seedlings were infected and transformation efficiency was measured by histochemical $\beta$-glucuronidase (GUS) staining (A) and methylumbelliferyl- $\beta$-glucuronide assay (B) at 3 days postinfection. $\mathbf{C}$ and $\mathbf{D}$, Efficiency of transient transformation using a root transformation assay. Root segments of 10-day-old Arabidopsis Col-0 and VirE2 and VirE3 constitutive expression lines were infected with either $10^{6}$ or $10^{7} \mathrm{CFU}$ of Agrobacterium cells per milliliter and transformation efficiency was measured by GUS staining (C) and percentage of root segments showing GUS activity (D) at 3 days postinoculation. $\mathbf{E}$ and $\mathbf{F}$, Efficiency of stable transformation using the root assay. Root segments of 10-dayold Arabidopsis Col-0, VirE2, and VirE3 constitutive expression plants were treated as in C and D, before transferring the segments to culture media for callus formation. All transformations employed Agrobacterium sp. strain GV3101 harboring the binary vector pBISN1 containing a GUS intron gene. Stable transformation efficiency was measured for callus tissue at 3-weeks postinoculation by GUS staining (E) and percentage of callus showing GUS activity (F). Significant differences in activity $(P<0.01)$ are indicated by double asterisks $(* *)$. 
well as cell division and development (Figs. 1D and 3). These results indicate that low concentrations of agrobacteria (such as at the early stage of infection) trigger only a very weak plant response, allowing easy infection of plant cells under natural conditions. Conversely, the higher concentration of agrobacteria causes stronger plant defense responses to the infection, as seen from the increased defense-gene expression induction and repression of development genes.

Also, by comparing the differences of plant gene-expression levels in response to avirulent and virulent Agrobacterium strains, we discovered that expression patterns of some genes were very different during the infection by these two strains. On one hand, expression of some genes was more highly induced during infection by avirulent than by virulent agrobacteria at earlier stages (Figs. 5 and 6). Because the difference between these two Agrobacterium strains is the presence or absence of a Ti plasmid, such a difference could be caused by some Vir proteins, such as components of the type IV secretion system, interacting with the plant cell at the beginning of the infection process for the virulent strain, as a result of coevolution of Agrobacterium tumefaciens with its host plant. On the other hand, effector-triggered immunity (ETI) causes a stronger global expression of both biotic and abiotic responsive genes than pathogen-associated molecular pattern-triggered immunity (PTI) at a later infection stage, indicated by the DEGs under virulent At804 versus avirulent A136 infection conditions as the infection progresses, regardless of up- or downregulation (Figs. 3, 5, and 6). Results from our GO enrichment analysis of upregulated genes in VirE3inducible expression lines argued for this speculation. These experiments showed that VirE2 and VirE3 overexpression lines had increased resistance to agrobacteria than that shown by wild-type plants. Our differential expression results differ slightly from results previously reported by Veena et al. (2003), who used suppression subtractive hybridization and DNA microarrays to analyze gene expression in response to Agrobacterium infection in tobacco cells grown in suspension culture. As these two separate studies employed different plant species and different plant culture systems for the transformation, it is not surprising that some differences in gene expression would be observed.

An important practical implication of our study using constitutive expression of VirE2 and VirE3 is that too-high concentrations of agrobacterium cells used for plant transformation under the laboratory conditions would trigger strong plant defense responses, compromising transformation results. Another interesting observation in our study was that constitutive expression of VirE2 or VirE3 increased plant resistance to Agrobacterium infection (Figs. 7 and 8), suggesting that this defense mechanism would enable plants to fight against pathogen attacks. Under natural infection conditions, Agrobacterium cell density is typically much lower than the lab experimental conditions and, therefore, rarely triggers a strong defense response whereas low concentrations of other pathogens can trigger defense response. This may reflect coevolution between Agrobacteriumplant interactions. Such a coevolutionary outcome as triggered by Vir proteins could favor Agrobacterium tumefaciens for their competitive niche over other pathogens. Upon infection by agrobacteria or other pathogens, plants employ different layers of defense responses, including PTI, which is first manifest in signals such as ROS burst or defense gene induction (Pitzschke et al. 2009a and b). A further response that may also be initiated is ETI, which is a general response seen in plants to Pseudomonas syringae pv. tomato DC3000 infection, characterized by programmed cell death of affected tissue (Abramovitch et al. 2003). Although a potential plant ETI response to Agrobacterium infection has yet to be characterized, our data here points to this possibility.

In summary, this study has elucidated some biological responses of plants to Agrobacterium colonization and the functions of some of the Vir proteins in plant defense. We have shown numerous genes that were highly responsive to the Agrobacterium-mediated transformation, for example, many defense- and stress-related genes were found to be upregulated, whereas genes associated with development and growth were down-regulated. Different responses of plant to avirulent or virulent Agrobacterium strains suggests that the T-DNA or Vir proteins play some roles in the plant gene expression. Constitutive expression of VirE2 or VirE3 induced plant defense responses and conferred to plants more resistance to the strains, suggesting a common defense pathway to bacterial pathogens and that Vir proteins have additional roles in enhanced plant defense responses in addition to directly facilitating T-DNA transformation. This not only contributes to our understanding of the molecular interaction between Agrobacterium tumefaciens and plants but, also, expands our knowledge about the roles of Vir proteins with respect to plant defense. Given the increasing identification of plant factors involved in this transformation process and the interactions of Agrobacterium effectors with plant proteins, a more complex picture of the signaling pathways involved in Agrobacterium-mediated transformation is emerging, as it becomes understood in detail.

\section{MATERIALS AND METHODS}

\section{Plant materials and transformation constructs.}

The Arabidopsis Col-0 ecotype and two Agrobacterium umefaciens strains (A136 and At804) were used in this study. These two strains are disarmed (lacking wild-type oncogenes within the T-DNA sequence). Thus, there are no plant-active hormones produced by either strain. Agrobacterium sp. strain A136 lacks a Ti plasmid, so it is incapable of transferring T-DNA or Vir proteins to plant cells. Strain At804 was derived from EHA105 and carries a disarmed supervirulent Ti plasmid and the binary vector pBISN1 (Gelvin 2003; Ni et al. 1995). pBISN1 has a gusA-intron gene, which can be expressed in plants but not in agrobacteria (Ni et al. 1995). This strain is able to transfer both T-DNA and Vir proteins into plant cells. To generate VirE2 or VirE3 constitutive transgenic plants, the VirE2 and VirE3 coding sequences were first PCR-amplified with Q5 highfidelity DNA polymerase (NEB), following manufacturer instructions, from Agrobacterium sp. strain C58 Ti plasmid with primers (Supplementary Table S2). The PCR reaction is as follows: denaturation $98^{\circ} \mathrm{C}$ for $30 \mathrm{~s}, 98^{\circ} \mathrm{C}$ for $10 \mathrm{~s}, 56^{\circ} \mathrm{C}$ for 10 to $30 \mathrm{~s}, 72^{\circ} \mathrm{C}$ for $30 \mathrm{~s}, 30$ cycles, final extension $72^{\circ} \mathrm{C}$ for $2 \mathrm{~min}$. The PCR product was then cloned into the pCR 2.1-TOPO TA cloning vector (Invitrogen) and then subcloned into the pEarleygate202 expression vector. Then, the constructs were transformed into Col-0 plants using the floral-dip method.

\section{Infection of whole Arabidopsis seedlings with different Agrobacterium strains.}

The AGROBEST infection assay was used, in this study, for transient gene expression and RNA-seq of whole Arabidopsis seedlings (Wu et al. 2014). To evaluate histochemical GUS staining and enzymatic activity, 10-day-old Arabidopsis seedlings were infected with $10^{8} \mathrm{CFU}$ of At804 per milliliter. At 48 and $72 \mathrm{hpi}$, seedlings were harvested and used for histochemical GUS staining and activity tests (Jefferson et al. 1987). For RNA-seq, 10-day-old Arabidopsis seedlings were mock-treated (cocultivation liquid media only) or treated with one of the two Agrobacterium strains. Samples were harvested at six different timepoints $(0,3,6,12,24$, and $48 \mathrm{hpi})$. Three replicates were performed for each treatment. The seedlings were harvested at each timepoint and were frozen in liquid nitrogen immediately and, in each treatment, all replicates were pooled together for 
RNA extraction as one biological replicate for RNA-seq. Two biological replicates were carried out for sequencing.

\section{Plant total RNA extraction and RNA-seq.}

Total RNA was extracted from infected Arabidopsis seedlings using Trizol reagent (Invitrogen). RNA samples were submitted to the University of Missouri DNA Core Facility, where library preparation and high-throughput sequencing services were performed.

\section{GO category enrichment and plotting.}

GO enrichment analysis of biological process terms was determined using the Gene Ontology Consortium online tool (The Gene Ontology Consortium 2015), using separate sets of DEGs that were either upregulated or downregulated at each timepoint during comparisons between two conditions. A set of selected GO categories was determined from those categories that were found to be significantly enriched at a 0.05 significance level among up- or downregulated genes at any given timepoint. Redundant functional categories were eliminated from this set. The resulting $P$ values from the enrichment tests at each timepoint for selected GO categories were log base $_{10}$-transformed. These data were then plotted in $\mathrm{R}$, using the packages gplots (Warnes et al. 2016) and RColorBrewer (Neuwirth 2014), with $\log _{10}(P$ value $)=5$ designated as the upper limit. Genes that fell into selected GO categories that were differentially expressed at a 0.05 significance level were plotted by their $\log _{2}$-transformed FPKM values across the different experimental treatments, using gplots. These values were scaled by row, using color coded lines to illustrate $z$ scores falling above or below the mean of each row.

\section{Scatterplots, Venn diagrams, and $\boldsymbol{k}$-means clustering.}

Scatterplots were generated by $\log _{2}$-transforming the FPKM values for each gene after filtering for genes with very low read counts. The Scatterplot3d R package (Ligges and Mächler 2003) was used to plot expression levels from all three treatments against each other. Venn diagrams were generated, using the online tool BioVenn, by pasting sets of DEGs in each comparison into the webpage (Hulsen et al. 2008). $k$-means clusters were generated by converting read count values for each DEG to FPKM in EdgeR and feeding them into the clustering function of the package TrinityRNAseq (Grabherr et al. 2011). Clusters were determined by the tree-cutting method.

\section{qRT-PCR.}

First-strand cDNA was synthesized from extracted mRNA using oligo dT primer and reverse transcription (Invitrogen). qRT-PCR was performed using a Bio-Rad CFX96 real-time system and iQ SYBR green supermix (Bio-Rad) with 40 cycles. Templates were normalized with Arabidopsis UBQ10. Three biological and technical replicates were performed. Data were analyzed by BioRad CFX Manager 2.0 software. The comparative cycle threshold method $(\Delta \Delta \mathrm{Ct})$ was used to obtain the relative fold change.

\section{Root assay.}

The root transformation assay was performed as described by Park et al. (2014). Agrobacterium tumefaciens GV3101 carrying pBISN1 was used. Roots from 10-day-old plants of Col-0 and VirE2 or VirE3 constitutive expression lines were cut into $0.5-\mathrm{mm}$ segments and were infected with $10^{6}$ and $10^{7} \mathrm{CFU} / \mathrm{ml}$ of agrobacteria separately. For the stable transformation assay, root segments were inoculated with agrobacteria for 3 days and were then transferred to callus induction media, as previously described (Zhu et al. 2003). After 3 weeks in tissue culture, roots were stained with $0.2 \mathrm{mg}$ of 5-bromo-4-chloro-3-indolyl glucuronide (X-Gluc) per liter for GUS staining at $37^{\circ} \mathrm{C}$ overnight. Then, the percentage of root segments with GUS activity was counted. Root segments from a pool of at least 30 Arabidopsis plants formed one independent replicate, and each of three independent replicates was inoculated.

\section{Measurement of ROS generation.}

ROS production was measured as previously described (Smith and Heese 2014). ROS activity was elicited with $50 \mathrm{nM}$ elf26 or Pseudomonas syringae pv. tomato DC3000. Twenty leaf discs from 4-week-old wild type and VirE2 or VirE3 constitutive expression lines, respectively, were used for each measurement. Luminescence was measured over $40 \mathrm{~min}$.

\section{Statistical analysis.}

Experimental design and mean comparisons among treatments. A complete random design was employed for the experiments, with three replicates comparing different treatments. The mean was presented as the average of three replicates \pm standard deviation. The difference between the two different treatments was separated by student $t$ test at a $P<0.01$ significant level.

Transcriptomic read processing, sequence alignment, and annotation. The read libraries resulting from sequencing were first cleaned for reads with low-quality sequencing scores, using Trimmomatic with standard options, as described for singleend sequence data by Bolger et al. (2014). Reads aligning to A. thaliana mtDNA or rDNA sequences (acquired from The Arabidopsis Information Resource [TAIR] and SILVA databases, respectively) were then removed from the sample libraries using the Bowtie2 (Langmead and Salzberg 2012) alignment tool. The cleaned reads were then mapped to the $A$. thaliana genome using Tophat version 2.0.13 with Bowtie2 (Kim et al. 2013), using the A. thaliana TAIR10 toplevel DNA FASTA file as the reference genome sequence and the GTF annotation file also available from TAIR (Berardini et al. 2015). This alignment allowed up to two nucleotide mismatches per read and designated minimum and maximum intron lengths of 40 and 2,000 nucleotides, respectively. The resulting BAM files, representing only reads aligning to a single sequence, were used to generate a table of raw read counts for each gene with the HTSeq htseq-count function (Anders et al. 2015)

Statistical analysis of differential gene expression. Raw read counts for each sample were used to carry out differential expression analysis, using the R package EdgeR (Robinson et al. 2010). Prior to analysis, genes showing a sum total of less than five read counts in at least five treatment-time designated samples were filtered out from the dataset. Read counts were normalized by library size and were then grouped by treatment (mock, avirulent, virulent) and time in a time series $(0,3,6,12,24$, and $48 \mathrm{hpi}$ ). Data were fit to a generalized linear model and common dispersion was estimated. A design matrix was constructed to account for time and treatment. Likelihood ratio tests were used to perform pairwise contrasts between each condition at the same timepoint. Genes with a false discovery rate of $<0.05$ (adjusted from $P$ value using Benjamini-Hochberg correction) were considered to show significant differential expression.

\section{ACKNOWLEDGMENTS}

We want to thank Z. Zhao and N. Wan for their technical assistance in laboratory work and greenhouse plant care, respectively. This work was supported by University of Missouri-Columbia Research Board (to K. Duan) and Life Sciences Fellowships (to C. Willig) funds.

\section{LITERATURE CITED}

Abramovitch, R. B., Kim, Y. J., Chen, S., Dickman, M. B., and Martin, G. B. 2003. Pseudomonas type III effector AvrPtoB induces plant 
disease susceptibility by inhibition of host programmed cell death EMBO J. 22:60-69.

Altpeter, F., Springer, N. M., Bartley, L. E., Blechl, A. E., Brutnell, T. P., Citovsky, V., Conrad, L. J., Gelvin, S. B., Jackson, D. P., Kausch, A. P., Lemaux, P. G., Medford, J. I., Orozco-Cárdenas, M. L., Tricoli, D. M., Van Eck, J., Voytas, D. F., Walbot, V., Wang, K., Zhang, Z. J., and Stewart, C. N., Jr. 2016. Advancing crop transformation in the era of genome editing. Plant Cell 28:1510-1520.

Anders, S., Pyl, P. T., and Huber, W. 2015. HTSeq-A Python framework to work with high-throughput sequencing data. Bioinformatics 31:166-169.

Azpiroz-Leehan, R., and Feldmann, K. A. 1997. T-DNA insertion mutagenesis in Arabidopsis: Going back and forth. Trends Genet. 13:152-156.

Ballas, N., and Citovsky, V. 1997. Nuclear localization signal binding protein from Arabidopsis mediates nuclear import of Agrobacterium VirD2 protein. Proc. Natl. Acad. Sci. U.S.A. 94:10723-10728.

Berardini, T. Z., Reiser, L., Li, D., Mezheritsky, Y., Muller, R., Strait, E., and Huala, E. 2015. The Arabidopsis information resource: Making and mining the "gold standard" annotated reference plant genome. Genesis 53:474-485.

Bolger, A. M., Lohse, M., and Usadel, B. 2014. Trimmomatic: A flexible trimmer for Illumina sequence data. Bioinformatics 30:2114-2120.

Cascales, E., and Christie, P. J. 2004. Definition of a bacterial type IV secretion pathway for a DNA substrate. Science 304:1170-1173.

Christie, P. J., and Vogel, J. P. 2000. Bacterial type IV secretion: Conjugation systems adapted to deliver effector molecules to host cells. Trends Microbiol. 8:354-360.

Citovsky, V., Warnick, D., and Zambryski, P. 1994. Nuclear import of Agrobacterium VirD2 and VirE2 proteins in maize and tobacco. Proc. Natl. Acad. Sci. U.S.A. 91:3210-3214.

Citovsky, V., Wong, M. L., and Zambryski, P. 1989. Cooperative interaction of Agrobacterium VirE2 protein with single-stranded DNA: Implications for the T-DNA transfer process. Proc. Natl. Acad. Sci. U.S.A. 86: 1193-1197.

Citovsky, V., Zupan, J., Warnick, D., and Zambryski, P. 1992. Nuclear localization of Agrobacterium VirE2 protein in plant cells. Science 256: 1802-1805.

Deng, W., Chen, L., Wood, D. W., Metcalfe, T., Liang, X., Gordon, M. P., Comai, L., and Nester, E. W. 1998. Agrobacterium VirD2 protein interacts with plant host cyclophilins. Proc. Natl. Acad. Sci. U.S.A. 95: 7040-7045

Ditt, R. F., Kerr, K. F., de Figueiredo, P., Delrow, J., Comai, L., and Nester, E. W. 2006. The Arabidopsis thaliana transcriptome in response to Agrobacterium tumefaciens. Mol. Plant-Microbe Interact 19:665-681.

Ditt, R. F., Nester, E. W., and Comai, L. 2001. Plant gene expression response to Agrobacterium tumefaciens. Proc. Natl. Acad. Sci. U.S.A. 98:10954-10959.

Djamei, A., Pitzschke, A., Nakagami, H., Rajh, I., and Hirt, H. 2007. Trojan horse strategy in Agrobacterium transformation: Abusing MAPK defense signaling. Science 318:453-456.

Escobar, M. A., and Dandekar, A. M. 2003. Agrobacterium tumefaciens as an agent of disease. Trends Plant Sci. 8:380-386.

García-Rodríguez, F. M., Schrammeijer, B., and Hooykaas, P. J. 2006. The Agrobacterium VirE3 effector protein: A potential plant transcriptional activator. Nucleic Acids Res. 34:6496-6504.

Gelvin, S. B. 1998. Agrobacterium VirE2 proteins can form a complex with $\mathrm{T}$ strands in the plant cytoplasm. J. Bacteriol. 180:4300-4302.

Gelvin, S. B. 2003. Improving plant genetic engineering by manipulating the host. Trends Biotechnol. 21:95-98.

Grabherr, M. G., Haas, B. J., Yassour, M., Levin, J. Z., Thompson, D. A., Amit, I., Adiconis, X., Fan, L., Raychowdhury, R., Zeng, Q., Chen, Z., Mauceli, E., Hacohen, N., Gnirke, A., Rhind, N., di Palma, F., Birren, B. W., Nusbaum, C., Lindblad-Toh, K., Friedman, N., and Regev, A. 2011. Full-length transcriptome assembly from RNA-Seq data without a reference genome. Nat. Biotechnol. 29:644-652.

Hansen, G. 2000. Evidence for Agrobacterium-induced apoptosis in maize cells. Mol. Plant-Microbe Interact 13:649-657.

Hulsen, T., de Vlieg, J., and Alkema, W. 2008. BioVenn-A web application for the comparison and visualization of biological lists using area-proportional Venn diagrams. BMC Genomics 9:488.

Hwang, H. H., and Gelvin, S. B. 2004. Plant proteins that interact with VirB2, the Agrobacterium tumefaciens pilin protein, mediate plant transformation. Plant Cell 16:3148-3167.

Jefferson, R. A., Kavanagh, T. A., and Bevan, M. W. 1987. GUS fusions: Beta-glucuronidase as a sensitive and versatile gene fusion marker in higher plants. EMBO J. 6:3901-3907.

Kim, D., Pertea, G., Trapnell, C., Pimentel, H., Kelley, R., and Salzberg, S. L. 2013. TopHat2: Accurate alignment of transcriptomes in the presence of insertions, deletions and gene fusions. Genome Biol. 14:R36.
Krastanova, S. V., Balaji, V., Holden, M. R., Sekiya, M., Xue, B., Momol, E. A., and Burr, T. J. 2010. Resistance to crown gall disease in transgenic grapevine rootstocks containing truncated virE2 of Agrobacterium. Transgenic Res. 19:949-958.

Lacroix, B., Vaidya, M., Tzfira, T., and Citovsky, V. 2005. The VirE3 protein of Agrobacterium mimics a host cell function required for plant genetic transformation. EMBO J. 24:428-437.

Langmead, B., and Salzberg, S. L. 2012. Fast gapped-read alignment with Bowtie 2. Nat. Methods 9:357-359.

Li, J., Krichevsky, A., Vaidya, M., Tzfira, T., and Citovsky, V. 2005. Uncoupling of the functions of the Arabidopsis VIP1 protein in transient and stable plant genetic transformation by Agrobacterium. Proc. Natl. Acad. Sci. U.S.A. 102:5733-5738.

Ligges, U., and Mächler, M. 2003. Scatterplot3d-an R package for visualizing multivariate data. J. Stat. Softw. 8:1-20.

Magori, S., and Citovsky, V. 2011. Agrobacterium counteracts host-induced degradation of its effector F-box protein. Sci. Signal. 4:ra69.

Mysore, K. S., Bassuner, B., Deng, X. B., Darbinian, N. S., Motchoulski, A., Ream, W., and Gelvin, S. B. 1998. Role of the Agrobacterium tumefaciens VirD2 protein in T-DNA transfer and integration. Mol. Plant-Microbe Interact 11:668-683.

Narasimhulu, S. B., Deng, X. B., Sarria, R., and Gelvin, S. B. 1996. Early transcription of Agrobacterium T-DNA genes in tobacco and maize. Plant Cell 8:873-886.

Neuwirth, E. 2014. RColorBrewer: ColorBrewer Palettes. R package version 1.1-2. The R Foundation.

Ni, M., Cui, D., Einstein, J., Narasimhulu, S., Claudia, E. V., and Gelvin, S. B. 1995. Strength and tissue specificity of chimeric promoters derived from the octopine and mannopine synthase genes. Plant J. 7:661-676.

Niu, X., Zhou, M., Henkel, C. V., van Heusden, G. P., and Hooykaas, P. J. 2015. The Agrobacterium tumefaciens virulence protein VirE3 is a transcriptional activator of the F-box gene VBF. Plant J. 84:914-924.

Park, S. Y., Yin, X., Duan, K., Gelvin, S. B., and Zhang, Z. J. 2014. Heat shock protein 90.1 plays a role in Agrobacterium-mediated plant transformation. Mol. Plant 7:1793-1796.

Pitzschke, A., Djamei, A., Teige, M., and Hirt, H. 2009a. VIP1 response elements mediate mitogen-activated protein kinase 3-induced stress gene expression. Proc. Natl. Acad. Sci. U.S.A. 106:18414-18419.

Pitzschke, A., Schikora, A., and Hirt, H. 2009b. MAPK cascade signalling networks in plant defence. Curr. Opin. Plant Biol. 12:421-426.

$\mathrm{Pu}, \mathrm{X}$. A., and Goodman, R. N. 1993. Attachment of agrobacteria to grape cells. Appl. Environ. Microbiol. 59:2572-2577.

Robinson, M. D., McCarthy, D. J., and Smyth, G. K. 2010. edgeR: A Bioconductor package for differential expression analysis of digital gene expression data. Bioinformatics 26:139-140.

Schrammeijer, B., den Dulk-Ras, A., Vergunst, A. C., Jurado Jácome, E., and Hooykaas, P. J. 2003. Analysis of Vir protein translocation from Agrobacterium tumefaciens using Saccharomyces cerevisiae as a model: Evidence for transport of a novel effector protein VirE3. Nucleic Acids Res. 31:860-868.

Smith, E. F., and Townsend, C. O. 1907. A plant-tumor of bacterial origin. Science 25:671-673.

Smith, J. M., and Heese, A. 2014. Rapid bioassay to measure early reactive oxygen species production in Arabidopsis leave tissue in response to living Pseudomonas syringae. Plant Methods 10:6.

The Gene Ontology Consortium. 2015. Gene Ontology Consortium: Going forward. Nucleic Acids Res. 43:D1049-D1056.

Tzfira, T., Vaidya, M., and Citovsky, V. 2001. VIP1, an Arabidopsis protein that interacts with Agrobacterium VirE2, is involved in VirE2 nuclear import and Agrobacterium infectivity. EMBO J. 20:3596-3607.

Tzfira, T., Vaidya, M., and Citovsky, V. 2004. Involvement of targeted proteolysis in plant genetic transformation by Agrobacterium. Nature 431:87-92.

Veena, Jiang, H., Doerge, R. W., and Gelvin, S. B. 2003. Transfer of T-DNA and Vir proteins to plant cells by Agrobacterium tumefaciens induces expression of host genes involved in mediating transformation and suppresses host defense gene expression. Plant J. 35:219-236.

Vergunst, A. C., Schrammeijer, B., den Dulk-Ras, A., de Vlaam, C. M., Regensburg-Tuïnk, T. J., and Hooykaas, P. J. 2000. VirB/D4-dependent protein translocation from Agrobacterium into plant cells. Science 290: 979-982.

Warnes, G.R., Bolker, B., Bonebakker, L. Gentleman, R., Liaw, W. H. A., Lumley, T., Maechler, M., Magnusson, A., Moeller, S., Schwartz, M., and Venables, B. 2016. gplots: Various R programming tools for plotting data. $\mathrm{R}$ package version 3.2.3. The R Foundation, Boston. Published online.

Wood, D. W., Setubal, J. C., Kaul, R., Monks, D. E., Kitajima, J. P., Okura, V. K., Zhou, Y., Chen, L., Wood, G. E., Almeida, N. F., Jr., Woo, L., Chen, Y., Paulsen, I. T., Eisen, J. A., Karp, P. D., Bovee, D., Sr., 
Chapman, P., Clendenning, J., Deatherage, G., Gillet, W., Grant, C., Kutyavin, T., Levy, R., Li, M. J., McClelland, E., Palmieri, A., Raymond, C., Rouse, G., Saenphimmachak, C., Wu, Z., Romero, P., Gordon, D., Zhang, S., Yoo, H., Tao, Y., Biddle, P., Jung, M., Krespan, W., Perry, M., Gordon-Kamm, B., Liao, L., Kim, S., Hendrick, C., Zhao, Z. Y., Dolan, M., Chumley, F., Tingey, S. V., Tomb, J. F., Gordon, M. P., Olson, M. V., and Nester, E. W. 2001. The genome of the natural genetic engineer Agrobacterium tumefaciens C58. Science 294:2317-2323.

Wu, H. Y., Liu, K. H., Wang, Y. C., Wu, J. F., Chiu, W. L., Chen, C. Y., Wu, S. H., Sheen, J., and Lai, E. M. 2014. AGROBEST: An efficient Agrobacterium-mediated transient expression method for versatile gene function analyses in Arabidopsis seedlings. Plant Methods 10:19.
Zhu, Y., Nam, J., Humara, J. M., Mysore, K. S., Lee, L. Y., Cao, H., Valentine, L., Li, J., Kaiser, A. D., Kopecky, A. L., Hwang, H. H., Bhattacharjee, S., Rao, P. K., Tzfira, T., Rajagopal, J., Yi, H., Veena, Yadav, B. S., Crane, Y. M., Lin, K., Larcher, Y., Gelvin, M. J., Knue, M., Ramos, C., Zhao, X., Davis, S. J., Kim, S. I., Ranjith-Kumar, C. T., Choi, Y. J., Hallan, V. K., Chattopadhyay, S., Sui, X., Ziemienowicz, A., Matthysse, A. G., Citovsky, V., Hohn, B., and Gelvin, S. B. 2003. Identification of Arabidopsis rat mutants. Plant Physiol. 132:494-505.

Zipfel, C., Kunze, G., Chinchilla, D., Caniard, A., Jones, J. D., Boller, T., and Felix, G. 2006. Perception of the bacterial PAMP EF-Tu by the receptor EFR restricts Agrobacterium-mediated transformation. Cell 125:749-760. 